Bull. Fac.Agric., Cairo Univ., 70: 277-290 (2019).

\title{
AN ECONOMIC STUDY OF FOOD CONSUMPTION PATTERNS IN EGYPT
}

\author{
(Received:15.12.2019)
}

\author{
By \\ Moshera M.A.EL-Batran \\ The Higher Institute of Specific Studies, Giza, Egypt
}

\begin{abstract}
The pattern of food consumption is of the most important indicators of the consumption of different food commodities, and is an indicator of the relative distribution of consumption expenditure on different food commodities. So the study of consumption expenditure and its distribution are an important indicator to identify the pattern of income distribution and the level of consumption expenditure on different commodities, so as to identify the shifts in the spending behavior of different commodities needed by the family and consume; and useful in determining the relative importance of goods in both urban and rural Egypt. The main objective of this study was to identify the consumption expenditure patterns by estimating the expenditure elasticities of food groups in urban, rural and Egypt, and estimating the minimum expenditure on food groups in urban, rural and Egypt. The most important results were as follows:

- The average annual consumption expenditure for the family in urban Egypt in 2017 was about 71.956 thousand pounds, with a daily expenditure per capita of about 52 pounds, representing expenditure on food and drink for the family of which about $30.2 \%$, especially meat, dairy, cheese and eggs.

- The average annual household consumption expenditure for the rural Egypt in 2017 was about 62.296 thousands pounds, with a daily expenditure per capita of about 37 pounds, which represents expenditure on food and drink for the family, of which about $35.6 \%$, for meat and vegetables.

- The average annual household consumption expenditure for the Egypt in 2017 was about 76.194 thousands pounds, with a daily expenditure per capita of about 50 pounds, representing expenditure on food and drink for the family, of which about $29.1 \%$, for meat, dairy, cheese and eggs.

- Taking into account the devaluation of the purchasing power of the Egyptian pound (as a result of the devaluation of the pound against the dollar by about $45 \%$ in November 2016), it showed a slight improvement in urban consumer expenditure and its decline in the rural consumer.

- Expenditure elasticity of fish, food products not classified under other items, non-alcoholic beverages amounted to about $0.27,0.34$ and 0.22 , respectively, indicating that they are necessary commodities. The rest of the expenditure on food and drink in urban areas was almost necessary in 2017.

- The minimum expenditure for various food commodities in urban areas was about 7021.2 and 12857.6 pounds / year for 2015 and 2017, respectively.

- Expenditure elasticities of fish, fruit and food products not classified under other items amounted to about $0.45,0.15$ and 0.45 , respectively, indicating that they are necessary commodities.The rest of the expenditure on food and drink in the rural areas was almost necessary in 2017.

- The minimum expenditure for various food commodities in the rural areas was about 7,603,2 and 13,654.7 pounds / year for 2015 and 2017, respectively.

- Expenditure elasticities of fish and food products not classified under other items amounted to 0.36 and 0.37 respectively, indicating that they are essential goods. The remaining items of expenditure on food and drink of the Egypt was almost necessary in 2017.

- The minimum expenditure for various food commodities in Egypt was about 7,809.6 and 15,211.1 LE / year for 2015 and 2017, respectively.
\end{abstract}

Key words: an economic study, food consumption, Egypt 


\title{
دراسة اقتصادية لانماط الاستهلاك الغذائى فى مصر
}

\section{مشيرة محمد عبدالمجيد البطران}

المعهد العالى للدر اسات النوعية ـ الجيزة - مصر.

\begin{abstract}
ملخص

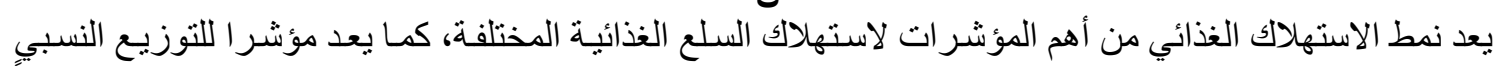

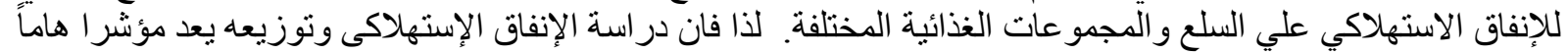

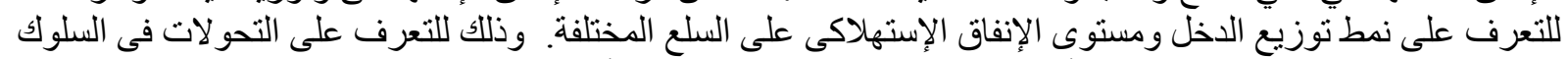

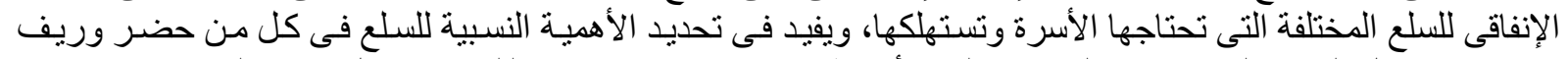

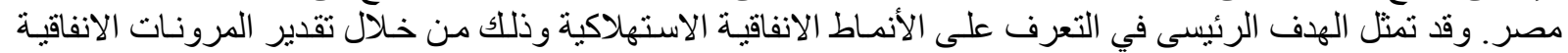

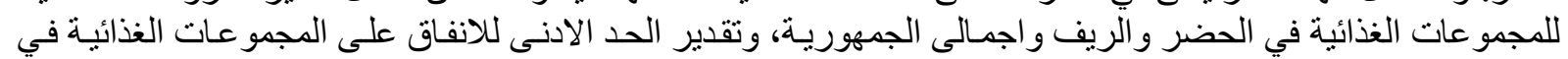

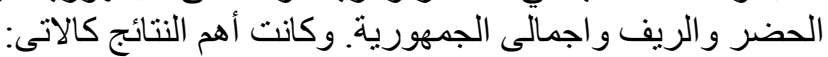

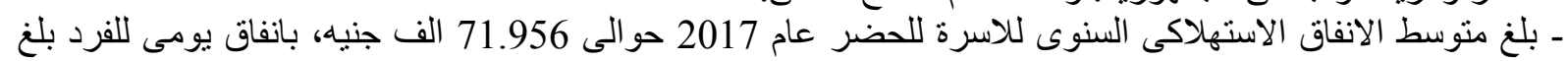

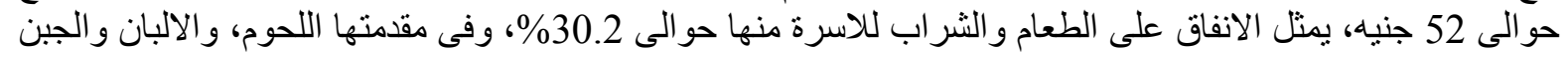
و البيض.

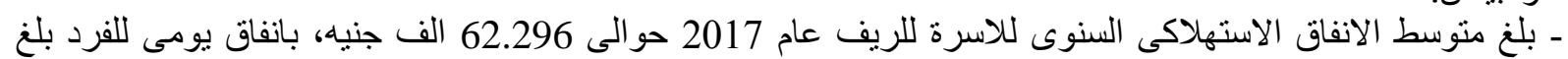

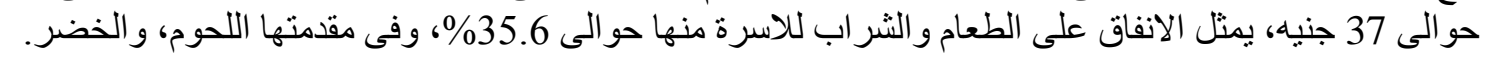

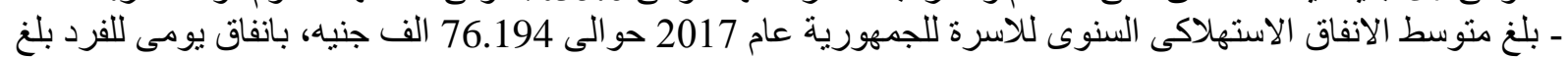

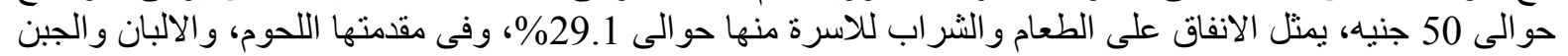

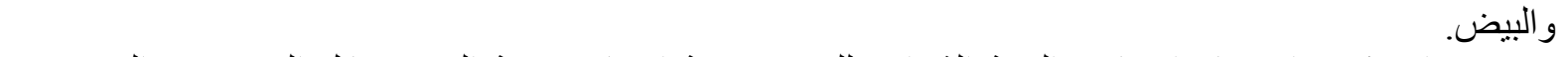

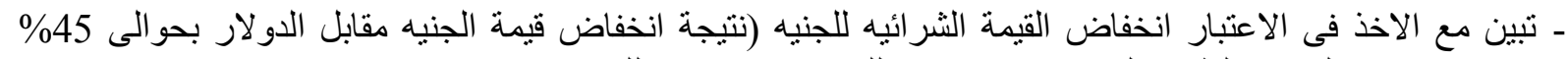

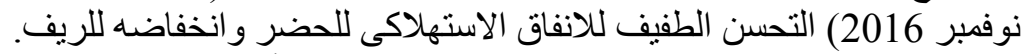

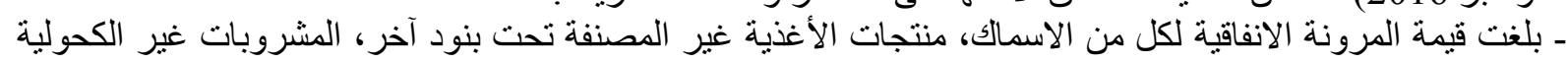

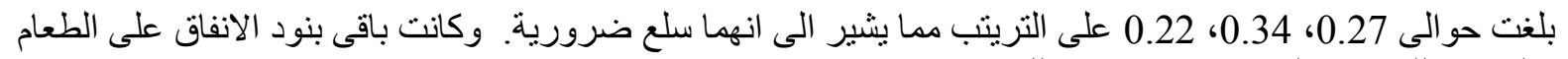

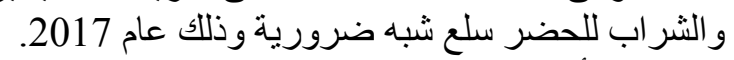

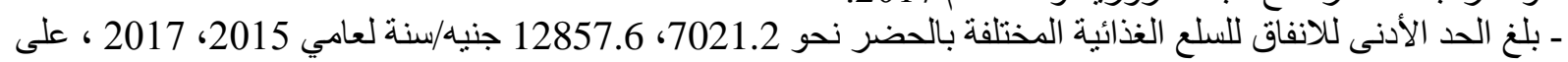

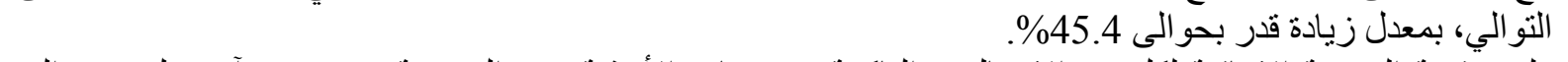

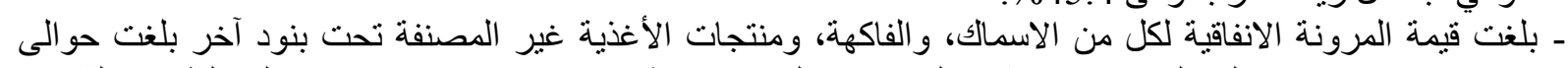

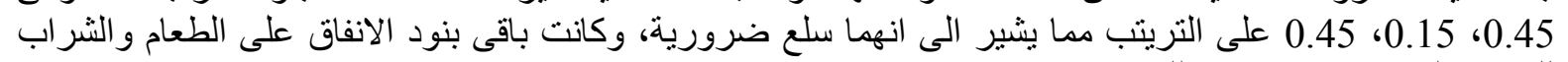
للريف سلع شبه ضرورية وذلك اللك عام 2017.

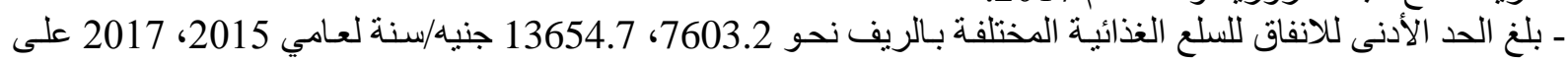

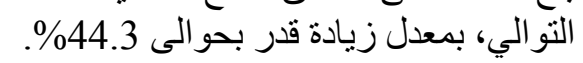

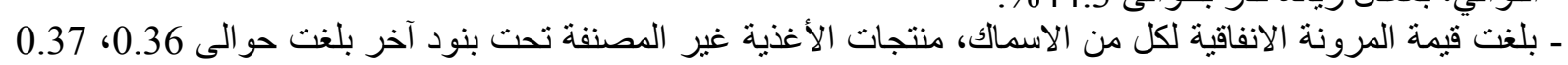

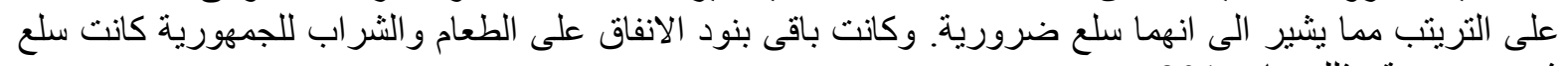

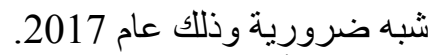
- بلغ الحد الأدنى للانفاق للسلع الغذائية المختلفة بالجمهوريـة نحو

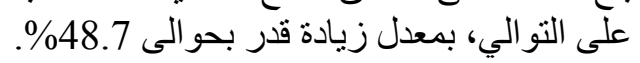

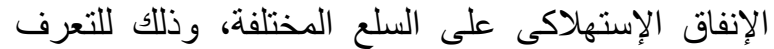

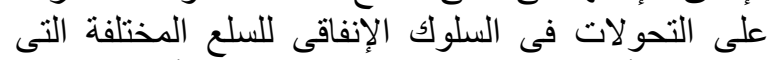

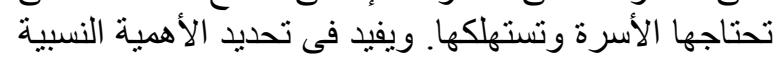

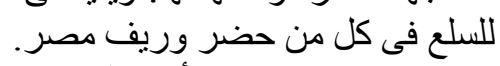

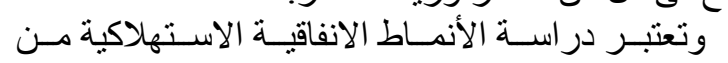

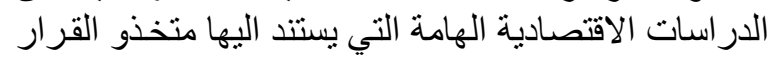

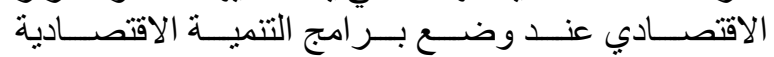

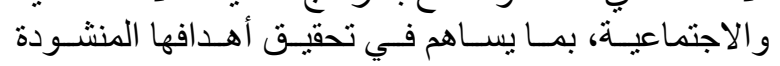

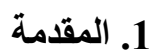

يعتبر الغذاء من المتطلبات الاساسية و الهامة

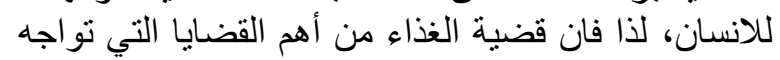

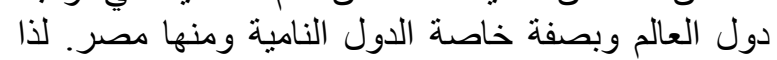

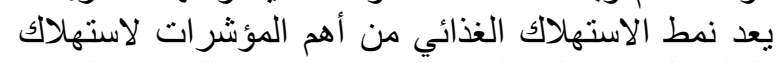

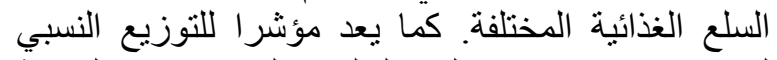

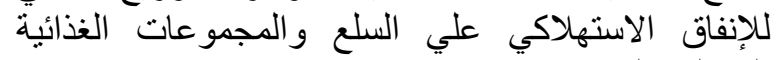

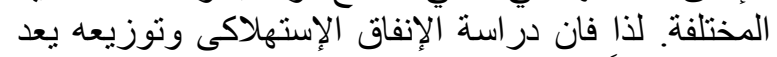

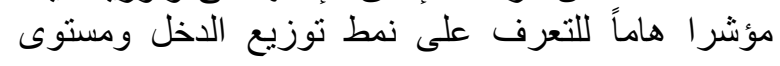


خلال فترة زمنية قصيرة المدى يفترض فيها عدم تغير

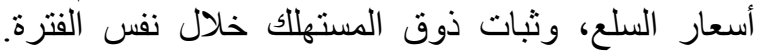

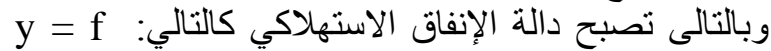

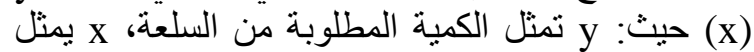

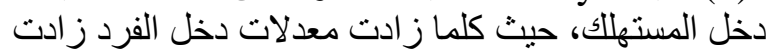

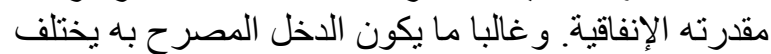

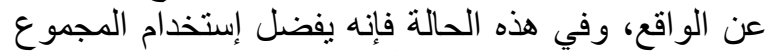

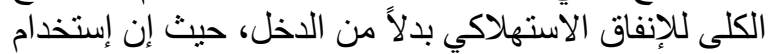

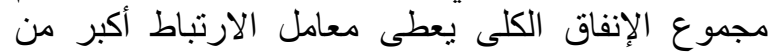

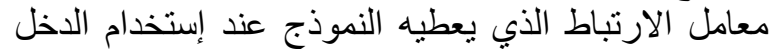

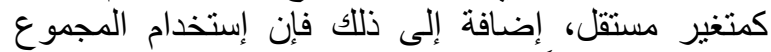

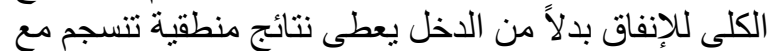

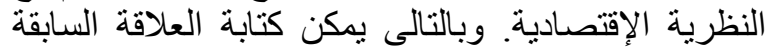
بالثكل التالي: yit

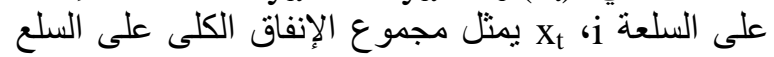

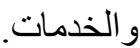

ويمكن وصف تحليل الطلب Seale, Regmi and ) Bernstein, 2003) التفضيلات بين مختلف السلع و الخدمات. وحيث انل الن الطلب على أبي سلعة أو مجمو عة من السلع يعتمد على التئ الاسعار

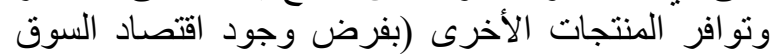

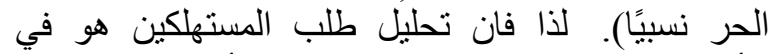
الأساس تحليل لتفضيلات المستهلك، أبي كيف يختار

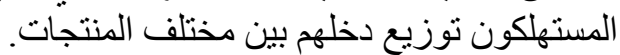

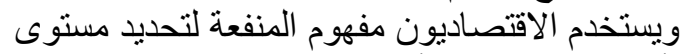

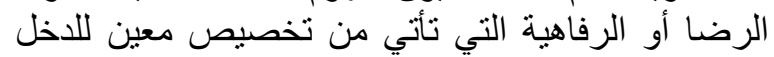

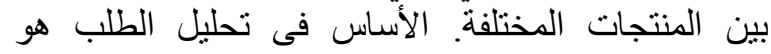

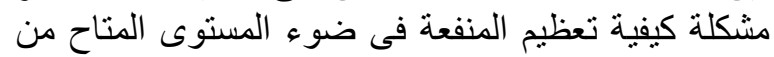

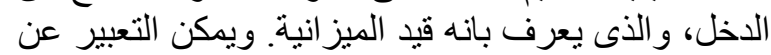

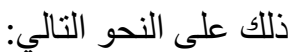
Maximize $\mathrm{u}=\mathrm{v}\left(\mathrm{q}_{1}, \mathrm{q}_{2}, . . \mathrm{q}_{\mathrm{n}}\right)$ subject to $\sum \mathrm{p}_{\mathrm{k}} \mathrm{q}_{\mathrm{k}}=x$,(1) حيث u دالة المنفة لكميات السلع المستهلكة، x هو إجمالي

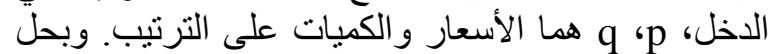

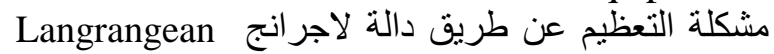
(Seale, Regmi and Bernstein, 2003) Fn إلى مجموعة من معادلات الطلب التي تعبر عن الكمية المطلوبة لكل سلعة كدالة فى السعر و إجمالي الدخل. $\mathrm{q}_{\mathrm{i}}=\mathrm{g}_{\mathrm{i}}(x, \mathrm{p})$,

حيث Pي متجه أسعار السلع. هذا النوع من دالة الطلب

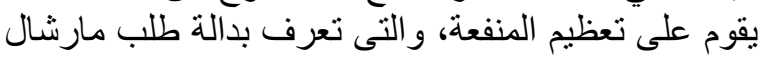
(Seale, Regmi and Bernstein, Marshallian uncompensated 2003 او غير التعويضية السعر التعويضية compensated تقيس استجابة الطلب

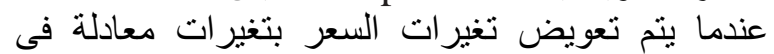

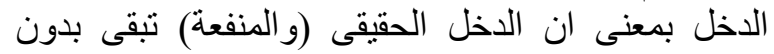

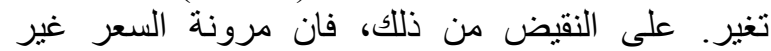

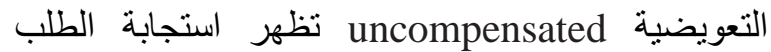

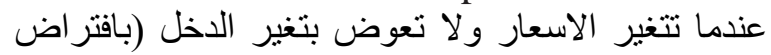

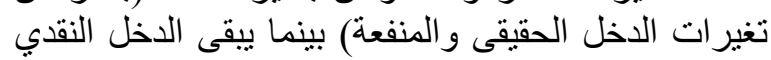

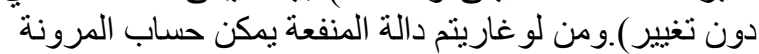

وبالتـالي رفع مسـتوي المعيشـة لكل أفر اد المجتمـع. كمــا

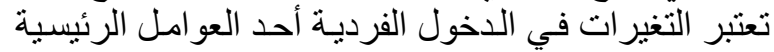
التي تؤثر على نمط إستهلاك المجير التمو عات السلعية المختلفة.

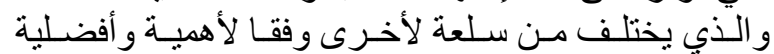

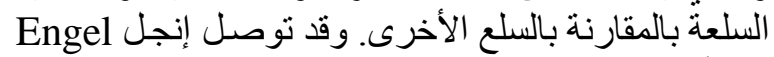

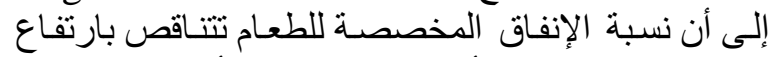

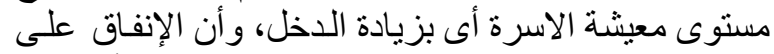

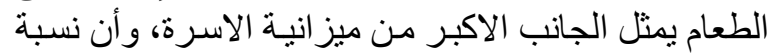

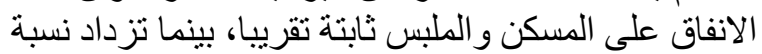

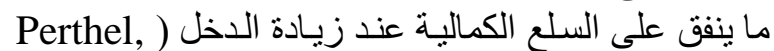

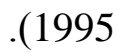

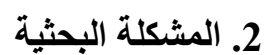

غالبا ماتحدث الكثير من التغير البثن في الاخدول الفردية

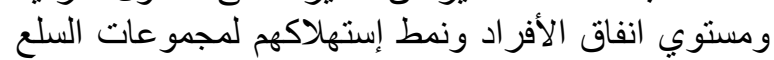

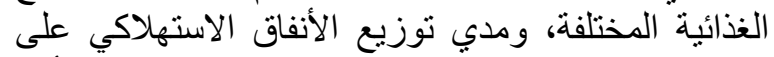

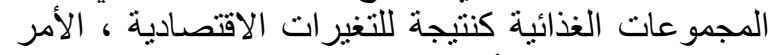

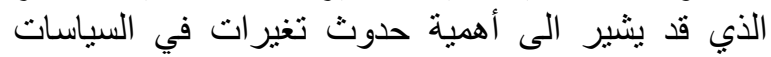

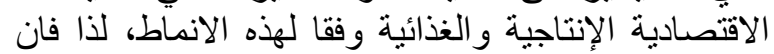

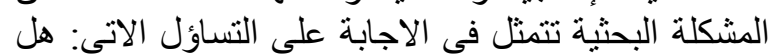

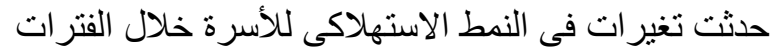

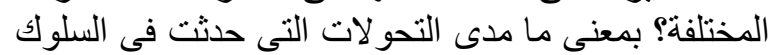

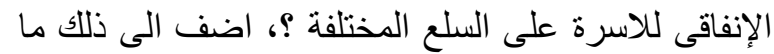

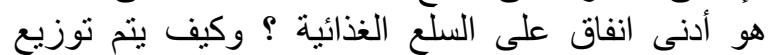
الفائض من الانفاق على السلع الغذائية؛

يعد تطور الانفاق الاستهلاكي البحثى الغذائي من الاهمية

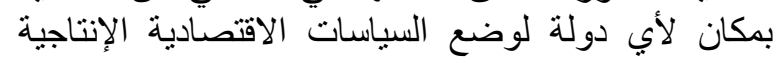

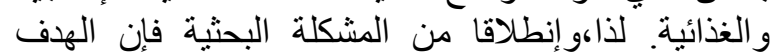

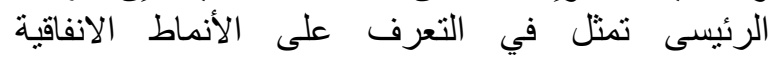
الاستهلاكية وذلك من في خلال تقدير المبلى المرونات الانفاقية

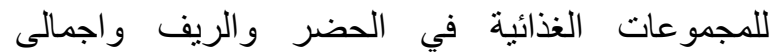

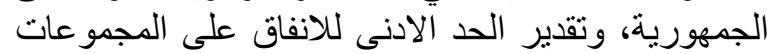
الغذائية في الحضر و الريف واجمالى الجمهورية.

\section{4. الطريقة البحثية ومصادر البيانات}

استخدم البحث في تحقيق أهدافه بيانات بحث البانئ الدخل

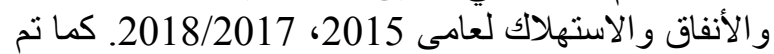
الاستعانة بالكتب والرسائل والبحوث لألثاث العلمية ذات الصلة بموضوع الأل باسنة. 4. 1. المنهجية البحثية المستخدمة

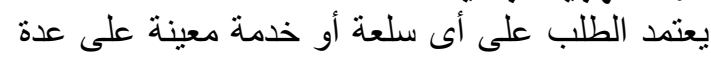

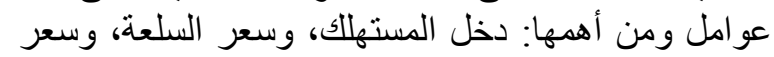

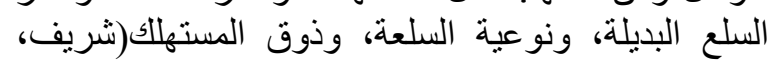
1981

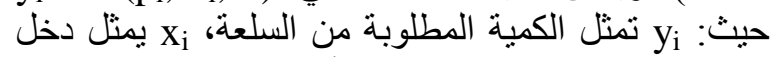

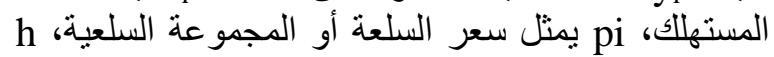
يمثل ذوق المستهلك. وحيث أن بحوث الدخل والإنفاق والاستهلاك تتفذ 
كنتيجة لتعظيم دالة المنفعة فى دالة طلب مارشال، أوتدنية التكاليف في دالة الطلب الهكسية. تمثل قيود الإضافة والتجانس التهية و التماتل و السلبية القيود

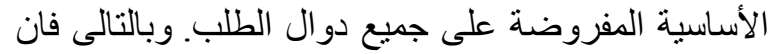

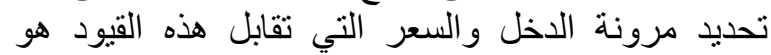
الهدف الرئيسي لتحليل الطلب. من الممكن تحديد مرونة

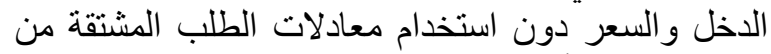

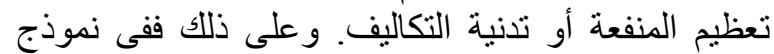

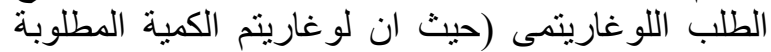

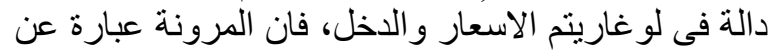

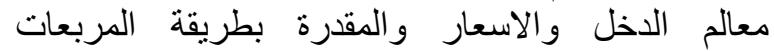
الصغرى العادية OLS لبيانات مقطعية أو سلاسل زمنية الصنية. وبالرغم من ان تعظيم المنفعة وتلبية القية القيود اللازمة التية،

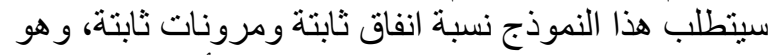
ما يتعارض مع الملاحظات التي نثير النئ إلى أن نسبة الانفاق تتغير عند تغير ات الدخل

Deaton and Muellbauer,(1984)

وعموما فان كلَّ من معادلات الطلب مارشال

و هيكسيان تلبى القيود المفروضة من قبل نظيل نظرية الطلب.

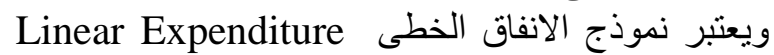
Stone and widely والمقدر من System (LES) لبيانات دولة واحدة سهل الاستخدام ويفترض تفنيل اضافية حيث يقيد الاستبدال بين السلع، كما بستبعد السلع

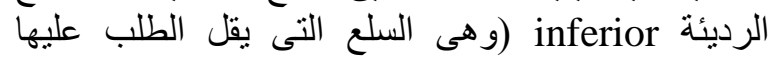

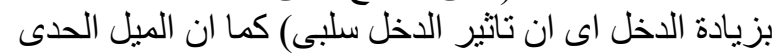

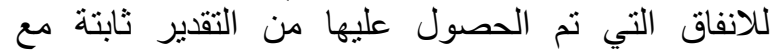

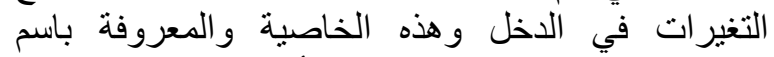

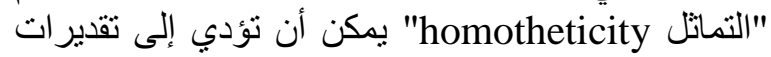

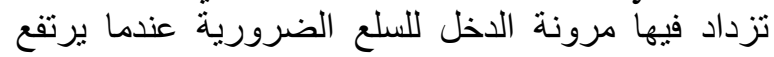
الدخل (Theil and Clements, 1987). ويستخدم نموذج روتردام Rotterdam و الذي

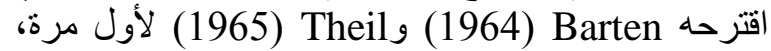

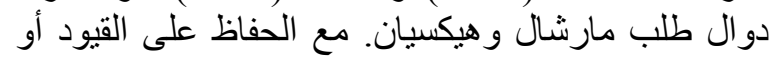

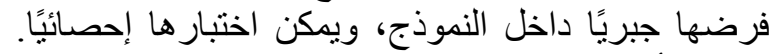

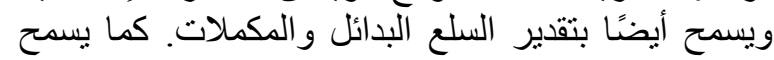
بفصل التفضيلات، و هي خاصية مر غوبة ومفيدة وليدة في تحليل

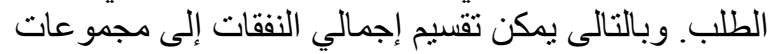

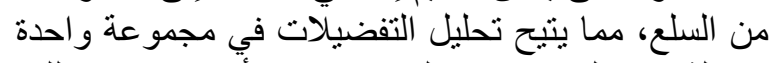

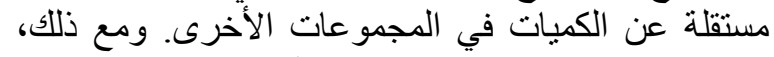

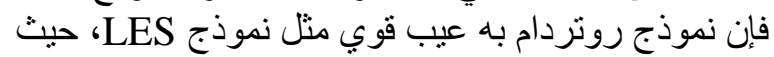

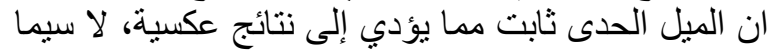

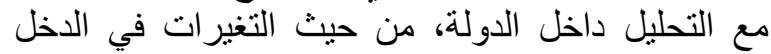

.(Barten, 1964 and Theil, 1965)

يتم تجنب مشكلة ثبات الميل الحدى للانفاق، وذلك الكي

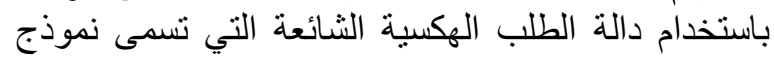
نظام الطلب شبه المثالي Almost Ideal Demand ن.System (AIDS) model

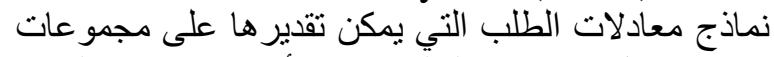

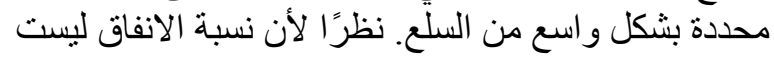

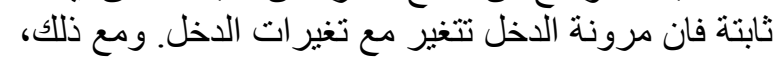

الدخلية والسعرية من تفاضل دالة لاجر انج كالاتى:

$d \log q_{i}=\eta_{i} d \log x+\sum_{j=1}^{n} \mu_{i j} d \log p_{j}$,

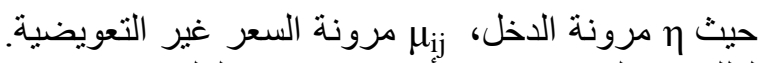

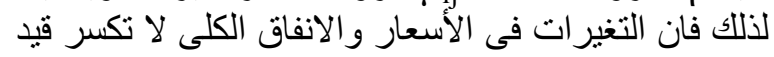

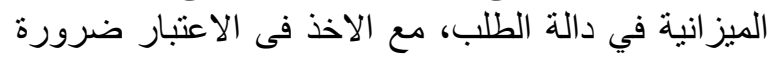
تحقق شروطَ مرونة التالية:

$\sum_{j=1}^{n} w_{j} \eta_{j}=1 \quad$ and $\quad \sum_{j=1}^{n} w_{i} \mu_{i j}+w_{j}=0$,

هيث هي نسبة الانفاق. ويعرف هذان الثرطان باسم تجميع إنجل وكورنو الانفي ويرن (Seale, Regmi and على الترتيب aggregation (Bernstein, 2003) ومعا في بعض الأحيان يشار إليها باسم قيد الإضافة.

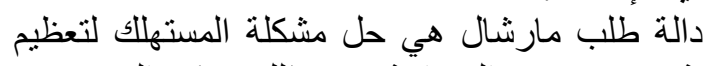

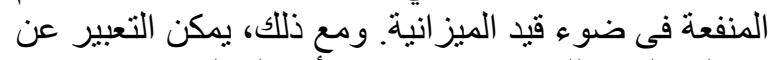

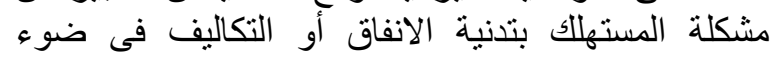
مستوى المنفعة المحدد سابقا كالاتى:

Maximize $x=\sum \mathrm{p}_{\mathrm{k}} \mathrm{q}_{\mathrm{k}}$ subject to $\mathrm{v}\left(\mathrm{q}_{1}, \mathrm{q}_{2}, . . \mathrm{q}_{\mathrm{n}}\right)=\mathrm{u}$. (5) الحل لهذه المشكلة هى دالة الطلب الهيكس Hicksian

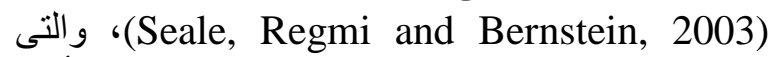
تعادل دالة طلب مارشال عند تقدير مسنوى المنفعة الأمثل $\mathrm{q}_{\mathrm{i}}=\mathrm{h}_{\mathrm{i}}(\mathrm{u}, \mathrm{P})=\mathrm{g}_{\mathrm{i}}(x, \mathrm{P})$ كالاتى دالة طلب هيكس Hicksian تعرف ايضا بدالة

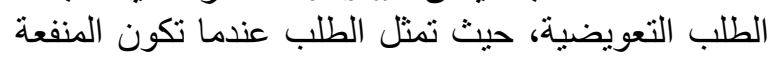

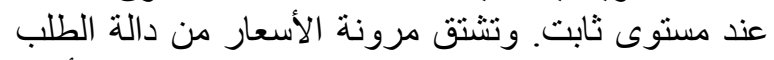

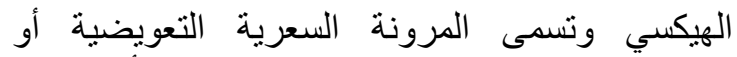

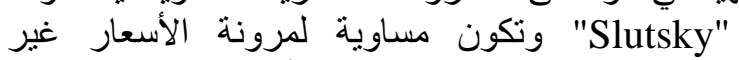

التعويضية (تسمى ايضا مرونة أسعار "Cournot") بالإضافة إلى مرونة الدخل ونسبة الانفاق اى:

$\varepsilon_{\mathrm{ij}}=\mathrm{u}_{\mathrm{ij}}+\eta_{\mathrm{i}} \mathrm{w}_{\mathrm{j}}$,

حيث

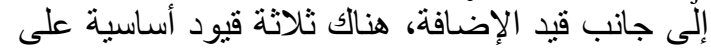

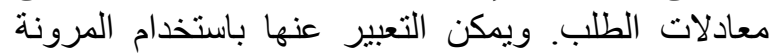
السعرية التعويضية على النحو التالي:

Homogeneity: $\sum_{j=1}^{n} \varepsilon_{i j}=0$

Symmetry: $\varepsilon_{\mathrm{ij}}=\varepsilon_{\mathrm{ji}}$

Negativity: $\sum_{i=1}^{n} \sum_{j=1}^{n} x_{i} w_{j} \varepsilon_{i j} x_{j}<0$,

for all $x_{\mathrm{i}}$ and $\mathrm{x}_{\mathrm{j}}$ that are not constants.

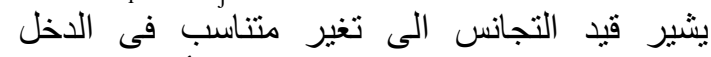

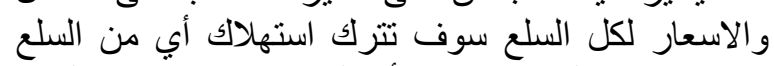

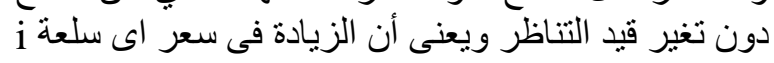

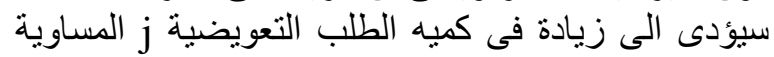

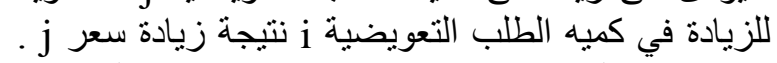

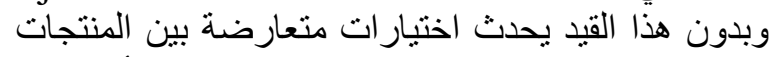

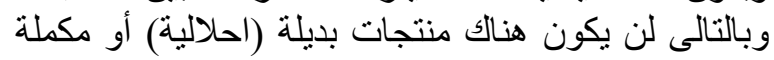
(تكاملية). اما قيد السلبية فياتى من تحدب دالة المالة المنفعة، 
وهى عبارة عن مجموعة من المعادلات و عددها

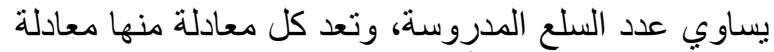

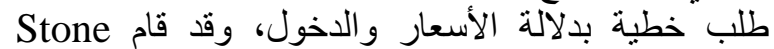
(1964) بقياس مستوى المعيشة اعتماد على دالة الألة المنفعة Stone-Geary utility function

Max:

$$
U=\sum_{j=1}^{n} \beta_{j} \ln \left(Q_{j}-\lambda_{j}\right), \quad \text { st: } \quad \sum_{j=1}^{n} P_{j} Q_{j}=Y
$$

ويتم الحصول على نموذج (LES) وذلكة الكئل عند تعظيم

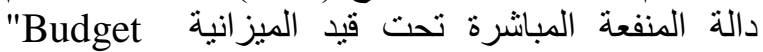
Constraint" "Singular Matrix" (XX)

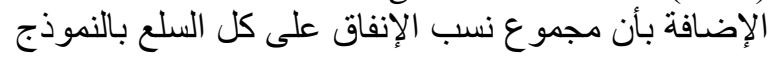

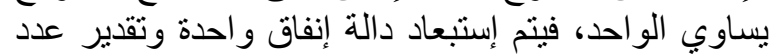
(n-1) $\mathrm{P}_{\mathrm{i}} \mathrm{Q}_{\mathrm{i}}=\mathrm{P}_{\mathrm{i}} \lambda_{\mathrm{i}}+\beta_{\mathrm{i}}\left(\mathrm{Y}-\sum_{\mathrm{j}=1} P_{\mathrm{j}} \lambda_{j}\right)(1)$

حيث: السلعة i Marginal Budget Share"

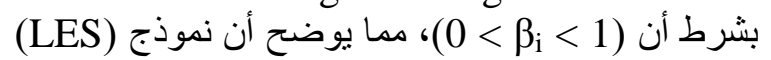
لا بسمح بوجود حالة سلع رديئة.

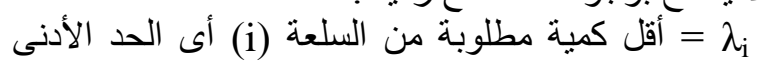

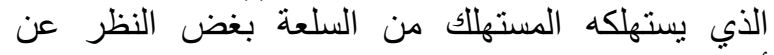
أسعار ها. الكفاف "Subsistence Expenditure دخل ز ائد منبق $=Y-\sum_{j=1}^{n} P_{j} \lambda_{j}$

(j) "Supernumerary Income"

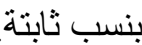
ويتضمن نموذج (LES) مرحلتين: الأولى نوضــــح

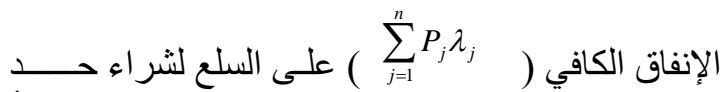

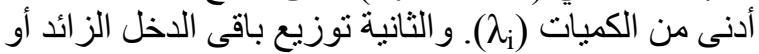
المتبقي (Yويتسم نموذج (LES) بأنه يستوفة في شروط التجانس

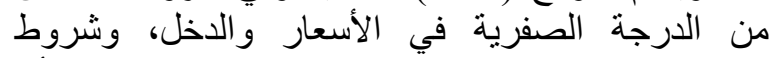

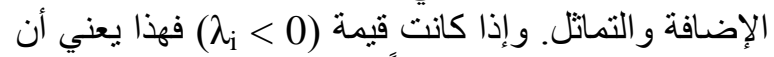
الطلب على السلعة يكون مرناً.

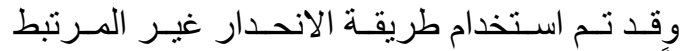

Seemingly Unrelated Regression ظاهريـاً Zellner's و التـي تُعرف أيضـاً بطريقة زيلنـر (SUR ) .Method

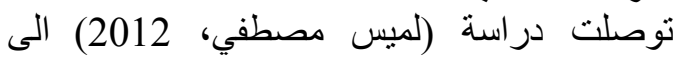

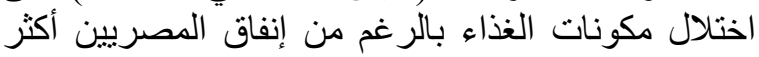

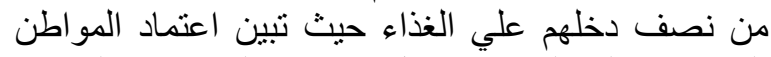

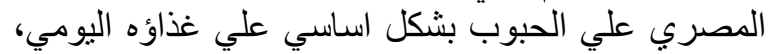

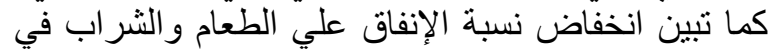

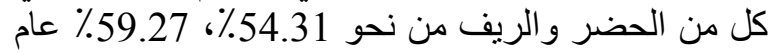

فإنه له العديد من العيوب، حيث المعلمات غير خطية

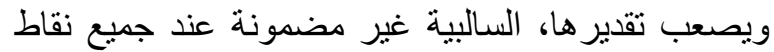

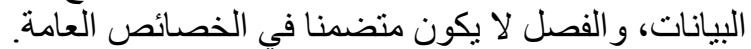

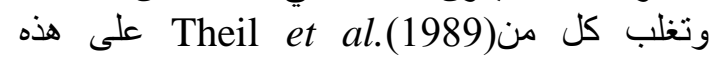

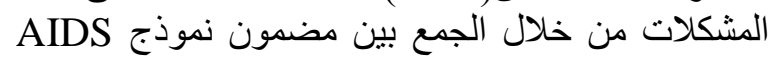
مع سمات الفصل في نموذج Rotterdam وسمى النموذج نموذج .Working's model لذا فقد نم استخدام كل من نموذج الإنج تقدير المرونات الانفاقية، ونموذج الإنفاق الخطي Expenditure System (LES الادنى للانفاق "حيث لا تصلح المقارنة بينهم لاختلاف

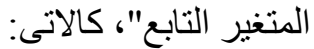

4. 1. 1. 1. نموذج النج . Seale et al.,(2003)

يعبر نموذج Working عن نسبة الانفاق كدالة خطية

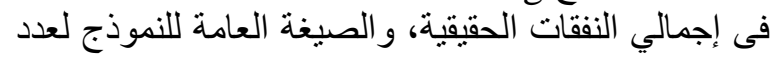

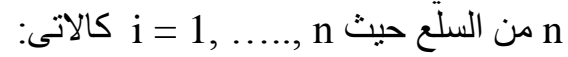
$\mathrm{w}_{\mathrm{i}}=\alpha_{\mathrm{i}}+\beta_{\mathrm{i}} \log \mathrm{E}+\varepsilon_{\mathrm{i}}$,

حيث Wi نسبة الانفاق على السلعة id E اجمالى الانفاق

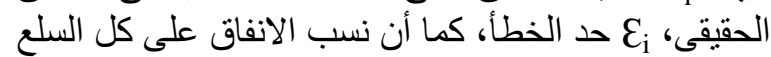
= 1 وذللك فى ضوء قديد الاضافة التالى:

$\sum_{i=1}^{n} \alpha_{i}=1$ and $\sum_{i=1}^{n} \beta_{i}=0$.

كما ان الميل الحدى للانفاق

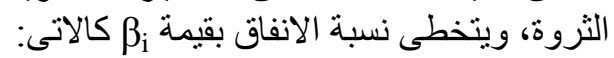

$\theta_{i}=\frac{d E_{i}}{d E}=\alpha_{i}+\beta_{i}(1+\log E)=w_{i}+\beta_{i}$.

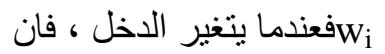

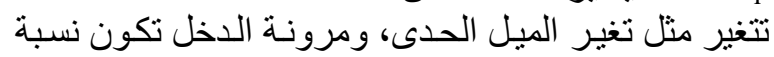

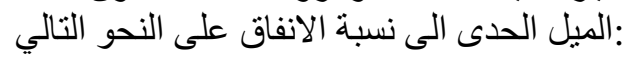

$\frac{\theta_{i}}{w_{i}}=\frac{d E_{i}}{d E} \frac{E}{E_{i}}=\frac{d\left(\log E_{i}\right)}{d(\log E)}$ وبقسمة المعادلة 13 على كالاتى:

$\frac{\theta_{i}}{w_{i}}=1+\frac{\beta_{i}}{w_{i}}$.

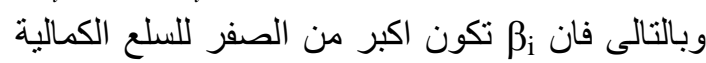

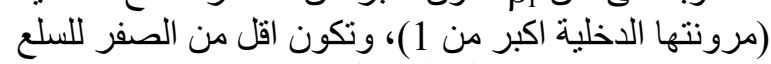

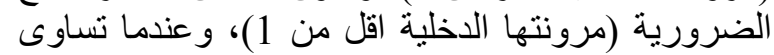

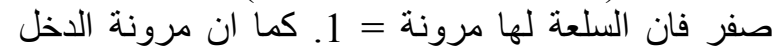

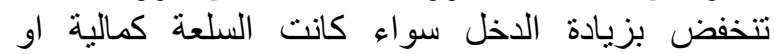

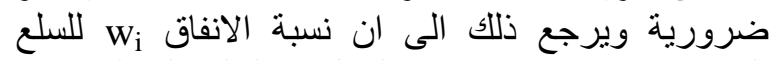

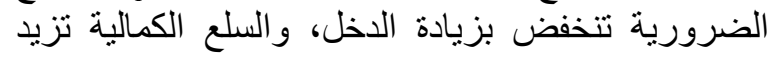

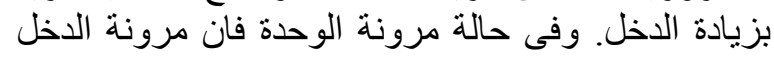
تبقى بدون تغيير مع تغير الدخل وذلك دلك لان لان نسبة الانفاق

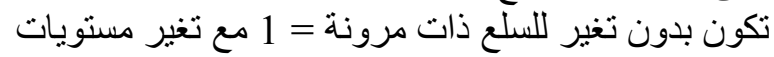

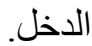

4. 1. 2. نموذج الإنفاق الخطي Linear :(Stone, 1964) Expenditure System (LES) 
الانفاق الكلى لكل المجمو عات الغذائية وغير الغذائية سواء

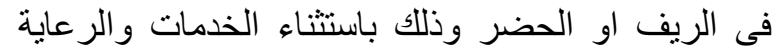

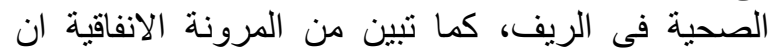
المجموعات الغذائية منخفضة الميفة المرونة مما يشير الى الى انها

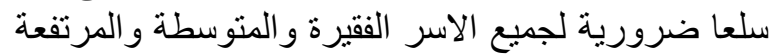

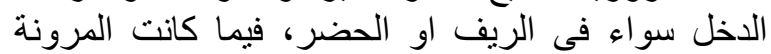

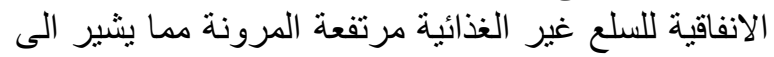

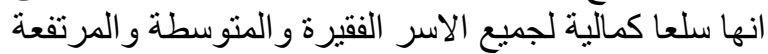
الدخل سو اء فى الريف او الحضر وذللك عام 2015.

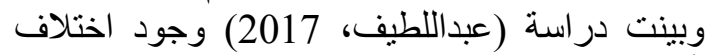

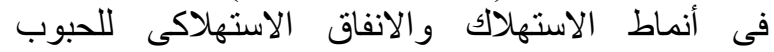

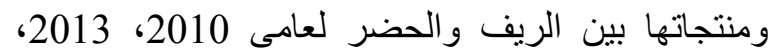

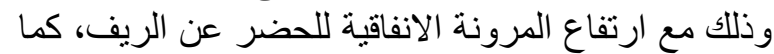
جاءت النتائج على مستوى الجمهورية مطابقة لنتائج الريف.

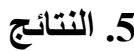

يتضح من جدول (1) ان متوسط الانفاق الاستهلاكى

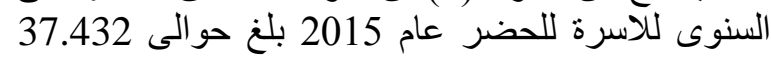

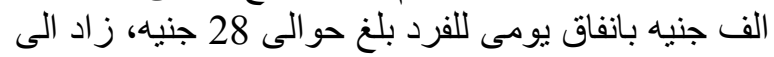

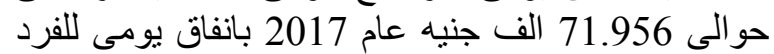
بلغ حوالى 52 جنيه، فى حين بلغ الانفاق على على الطعام

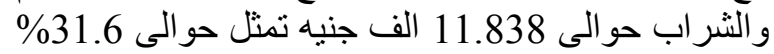
من إجمالى الانفاق الاستهلاكى عام 2015 زاد الى حوالى حوالى الى 21.705 الف جنيه نمثل حوالى الألى

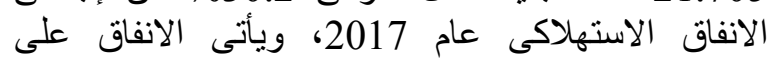

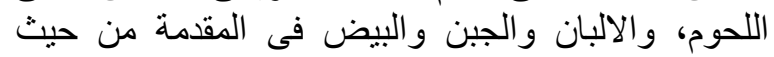

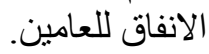

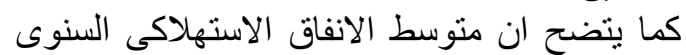

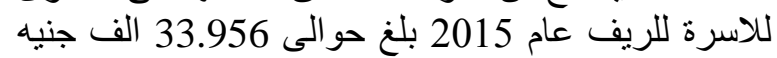

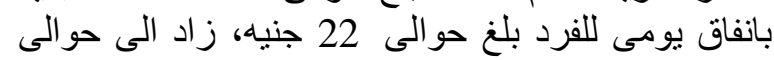

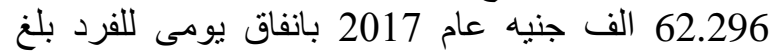
حوالى 37 جنيه، فى حين بلغ الانيه الانفاق على الطعام

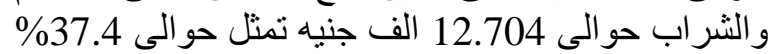
من إجمالى الانفاق الاستهلاكى عام 2015 زاد الى حوالى الى الى

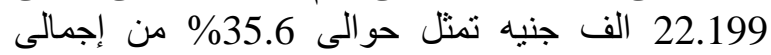

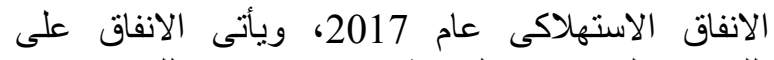
اللحوم، والخضر فى المقدمة من حيث الانفاق اللفان اللعامين.

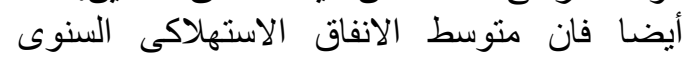

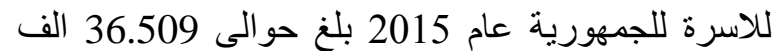

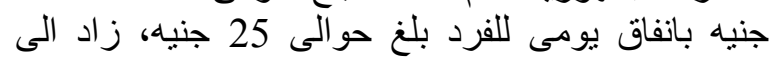

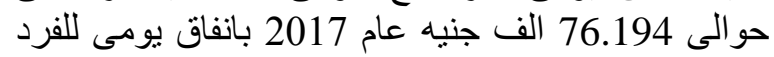

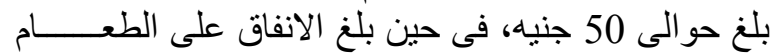

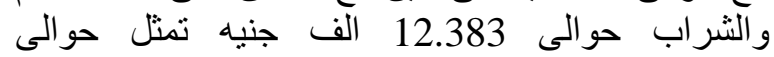

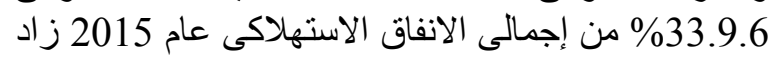

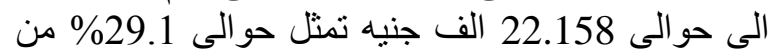

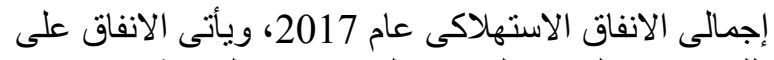

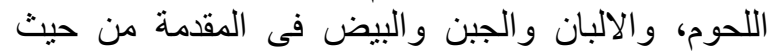

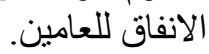

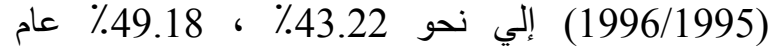

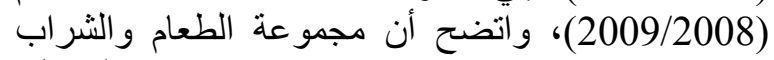

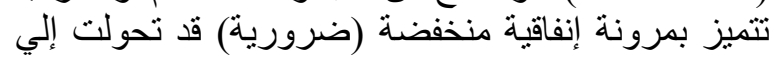

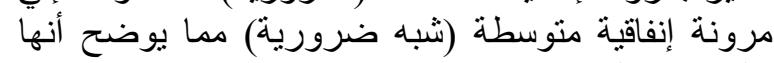

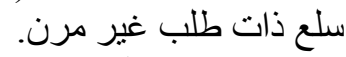

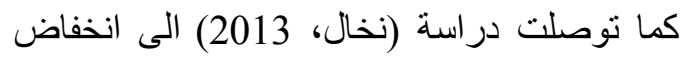
الأهية النسبية للإنفاق على السلع الاستهلاكية الغذائية لعام

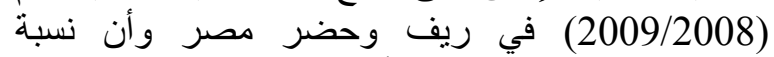
الانخفاض في الحضر كانت أكبر من نظبرتها في الريف،

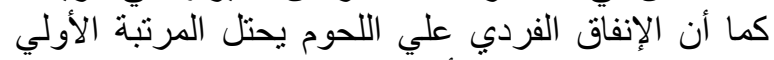

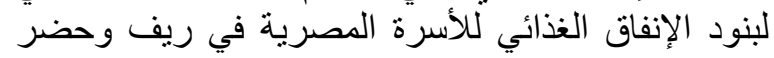

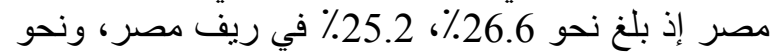

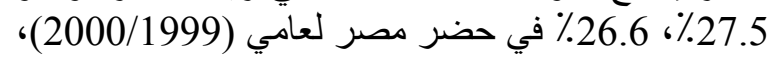
(2009/2008) علي الترتيب.

وبينت دراسة (هناء عبد اللطيف، 2014) 2014) أن معدل

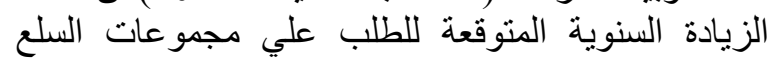

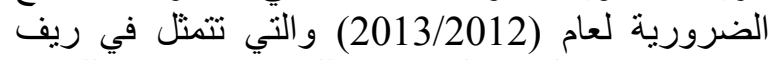

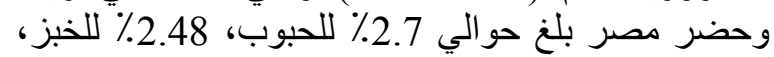

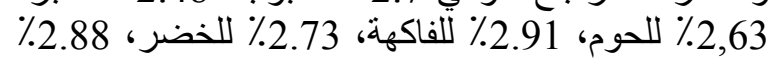
للسكر و الأغذية السكرية في الريف، 2.93\%، وحوالي 2.82.

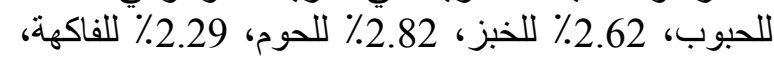

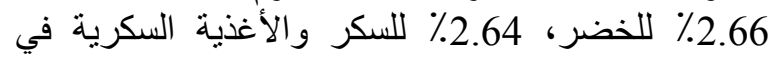

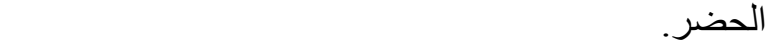
توصلت دراسة القبلاوي و هبة الله السيد،(2015) الى الى القي

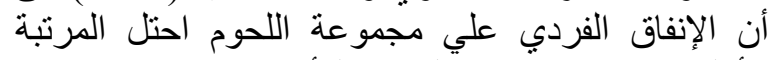
الأولي فى بنود الإنفاق الغذائي للأسرة في ريف اللغوم احتل وحضر النربة

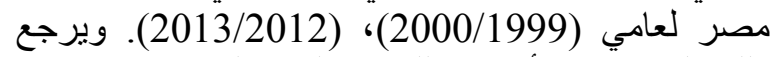
ذللك إلي ارتفاع أسعار اللحوم وليس إلي زيادة كمياتها

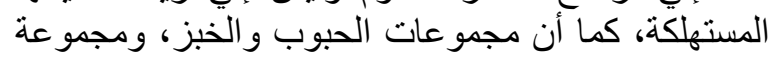

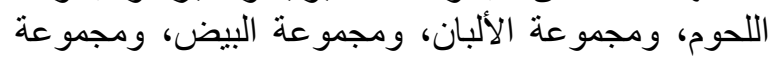

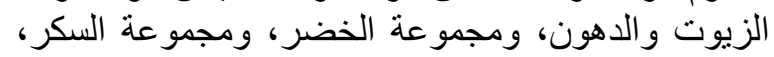

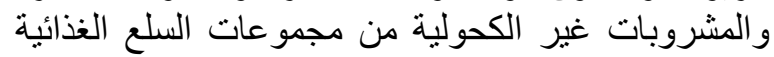

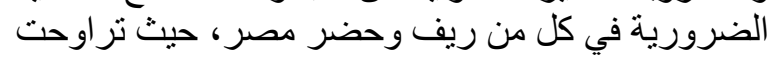

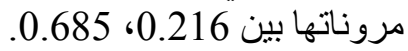

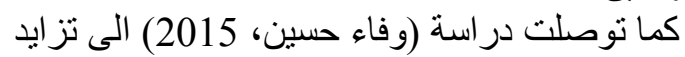

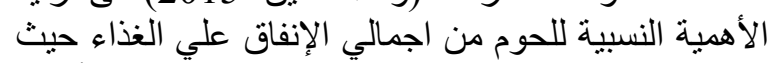

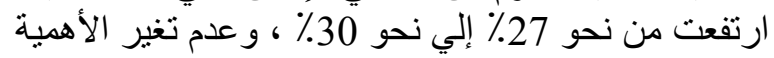

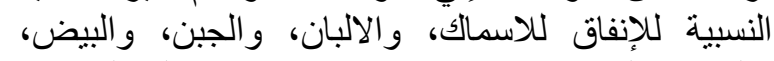

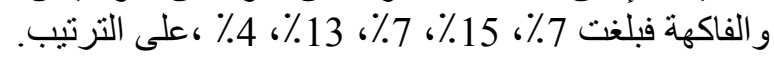

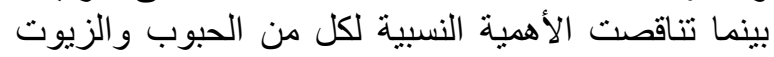

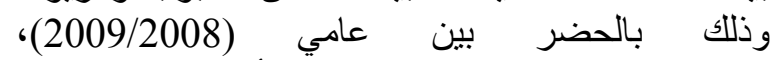

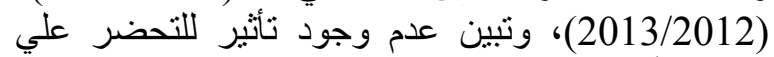

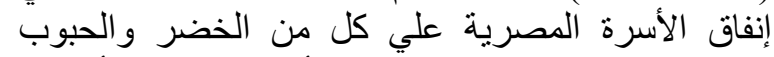

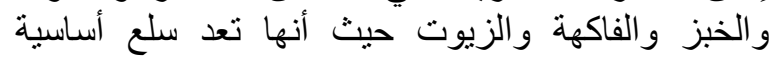

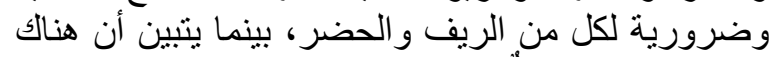

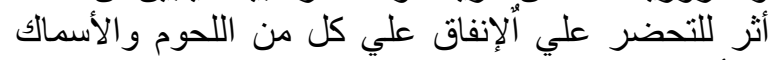
و الألبان و الجبن و البيض.

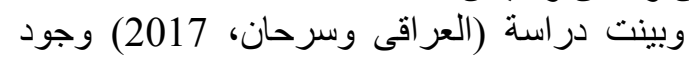

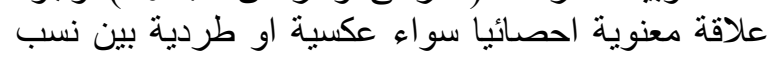
الانفاق على المجموعات السلعية المختلفة ولونية الوغاريتم 
جدول (1): متوسط الانفاق السنوى للاسرة على الغذاء فى الحضر والريف والجمهورية عامى 2015، 2017.

\begin{tabular}{|c|c|c|c|c|c|c|c|c|c|c|c|c|}
\hline \multicolumn{4}{|c|}{ جمهورية } & \multicolumn{4}{|c|}{ ريف } & \multicolumn{4}{|c|}{ حضر } & \multirow{3}{*}{ الإنفاق بنود } \\
\hline \multicolumn{2}{|c|}{2017} & \multicolumn{2}{|c|}{2015} & \multicolumn{2}{|c|}{2017} & \multicolumn{2}{|c|}{2015} & \multicolumn{2}{|c|}{2017} & \multicolumn{2}{|c|}{2015} & \\
\hline$\%$ & جنيه & $\%$ & جنيه & $\%$ & جنيه & $\%$ & جنيه & $\%$ & جنيه & $\%$ & جنيه & \\
\hline 12.5 & 2765.3 & 11.0 & 1359.3 & 12.8 & 2847.4 & 11.4 & 1446.2 & 12.1 & 2620.8 & 10.6 & 1252.5 & الحبوب والخيز \\
\hline 29.6 & 6568.9 & 30.3 & 3748.1 & 29.8 & 6615.0 & 30.8 & 3910.4 & 29.5 & 6409.7 & 30.0 & 3555.8 & 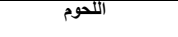 \\
\hline 6.8 & 1512.8 & 6.7 & 824.6 & 6.3 & 1393.4 & 6.2 & 792.2 & 7.2 & 1555.4 & 7.0 & 833.4 & |لاسماك \\
\hline 13.0 & 2886.0 & 13.8 & 1711.1 & 10.7 & 2380.3 & 12.0 & 1526.6 & 14.8 & 3219.1 & 15.7 & 1855.5 & الالبان والجين والبيض \\
\hline 8.1 & 1796.2 & 7.7 & 952.9 & 9.3 & 2056.2 & 8.6 & \begin{tabular}{|l|}
1094.1 \\
\end{tabular} & 7.3 & 1590.3 & 6.9 & 815.3 & الزيوت والدهون \\
\hline 6.0 & 1332.8 & 6.5 & 800.3 & 6.0 & 1328.3 & 6.4 & 814.7 & 6.0 & 1299.7 & 6.5 & 766.0 & الففاكها \\
\hline 12.5 & 2778.6 & 13.5 & 1674.4 & 13.7 & 3037.4 & 14.6 & 1852.0 & 11.8 & 2569.3 & 12.7 & 1501.7 & الخضر \\
\hline 5.1 & 1125.1 & 4.7 & 582.8 & 5.8 & 1284.2 & 4.7 & 602.3 & 4.5 & 980.1 & 4.5 & 528.7 & السكر والاغديه السكريه \\
\hline 1.9 & 424.7 & 2.0 & 247.5 & 1.9 & 427.1 & 2.0 & 251.0 & 1.9 & 420.7 & 2.0 & 237.7 & المصنفة تحت بند آخر \\
\hline 4.4 & 968.1 & 3.9 & 482.1 & 3.7 & 829.0 & 3.5 & 440.5 & 4.8 & 1039.5 & 4.2 & 492.1 & المثّروبات غير الكحوليه \\
\hline 29.1 & 22158.4 & 33.9 & 12383.1 & 35.6 & 22198.5 & 37.4 & 12703.7 & 30.2 & 21704.7 & 31.6 & 11837.7 & الطعام والثنر اب \\
\hline $4.2 / 50$ & 76193.8 & $4.0 / 25$ & 36509.3 & $4.6 / 37$ & 62296.4 & $4.3 / 22$ & 33956.2 & $3.8 / 52$ & 71955.6 & $3.6 / 28^{*}$ & 37432.0 & إلإسمالى الإكعاقى \\
\hline
\end{tabular}

ـ الفئات الانفاقية عام 2015 اقل من 10 الى اكثر من 100 الف جنيه، و عام 2017 اقل من 10 الى الى اكثر من 200 الف جنيها.

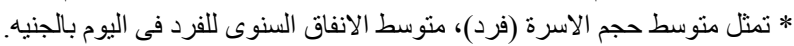

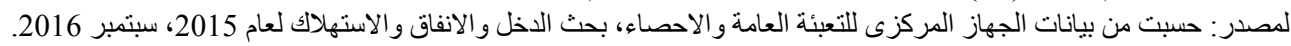

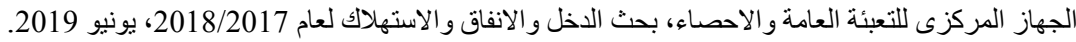

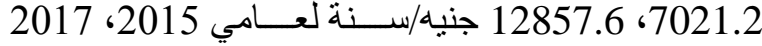
على التو الي، و الجزء المتبقي و الذي يعرف بالِ بالإنفاق الز ائد

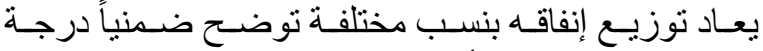

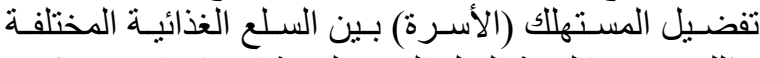
وذللك يتم وفقا لقيمة الميل الحدى لنسبة الإنفاق الاستهلاكي.

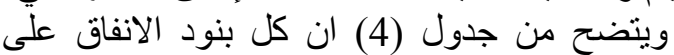

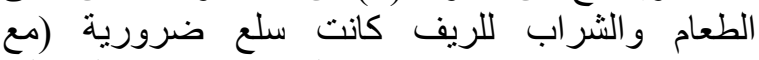

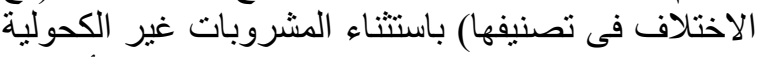

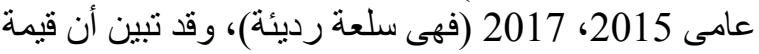

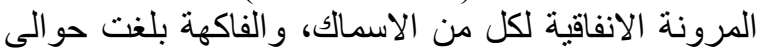

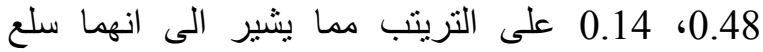
ضرورية، فى حين كانت باقى بنود الانفاق على التى الطعام

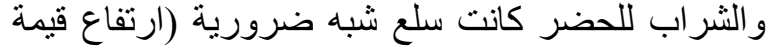
المرونة ولكنها لاز الت اقل من الو احد) وذلك علك عام 2015. كما تبين أن قيمة المرونة الانفاقية لكل من الاسماك، الاكي،

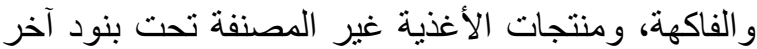
بلغت حو الى 0.45، 0.15، الى انهما سلع ضرورية، في حين كانت باقى بلى بنود الانفاق

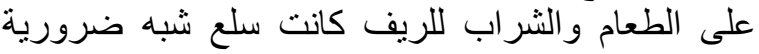

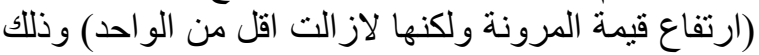

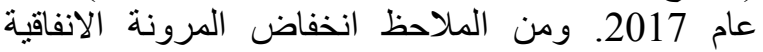

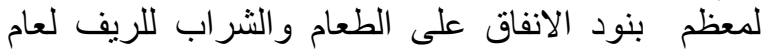

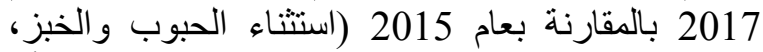
و الفاكهة، و السكر و الاغذية السكرية) مما قد يشير الى أن أن النئ هذه السلع أصبحت أكثر ضرورية.

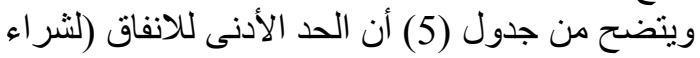

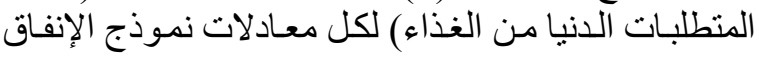

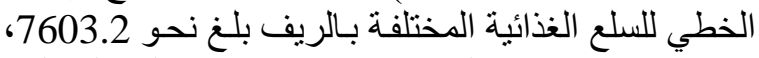

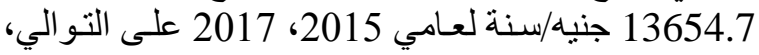

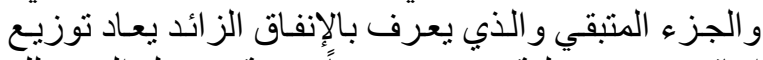

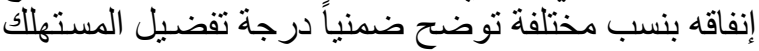
(الأسرة) بين السلع الغذائية المختلفة وذلك ينم وفقا لقيمة
ومن ذللك بتضح انخفاض نسبة الانفاق على الطعام

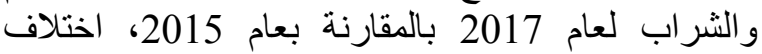

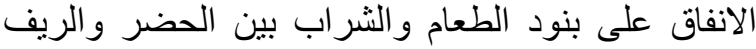

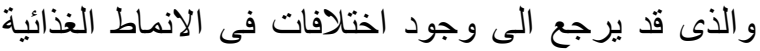

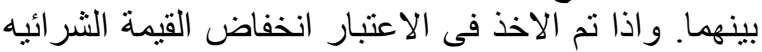

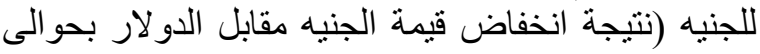

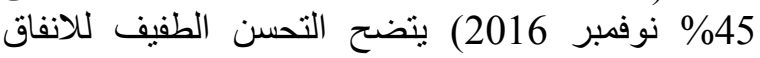
الاستهلاكى للحضر و وانخفاضه للريف.

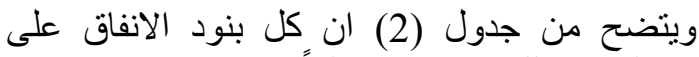

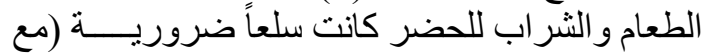

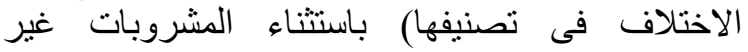

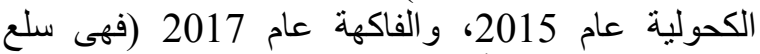
رديئة)، وقد تبين أن قيمة المرونة الانفاقية لكل من العن

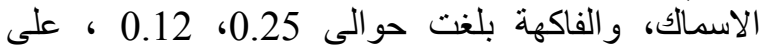

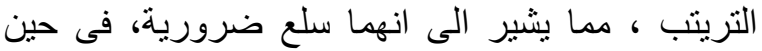

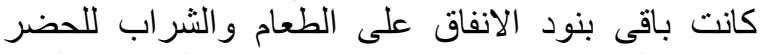

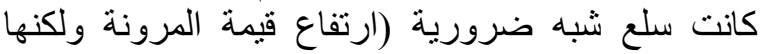

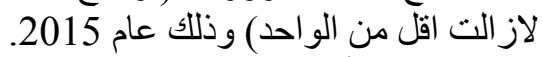
كما تبين أن قيمة المرونة الانفاقية لكل من الاسماك،

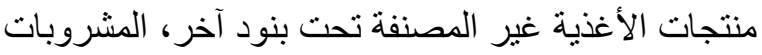

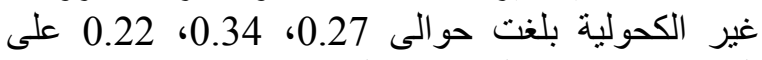

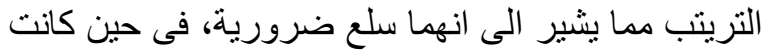

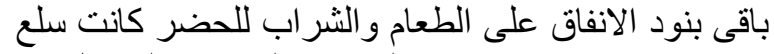

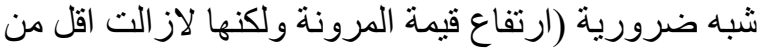

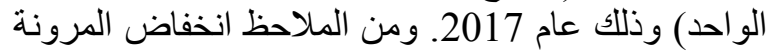

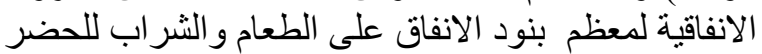

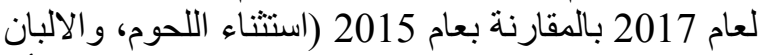

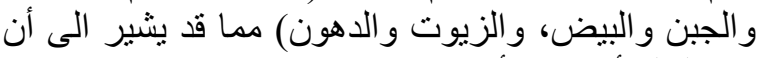
هذه السلع أصبحت أكثر ضرورية النية.

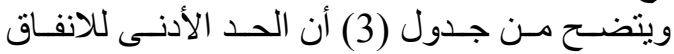

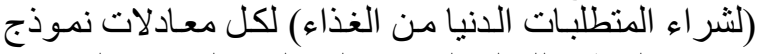

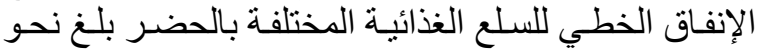




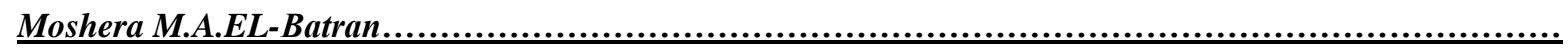

جدول (2): نتائج نموذج working لبنود الانفاق الغذائية للحضر لعام 2015، 2015.

\begin{tabular}{|c|c|c|c|c|c|c|c|c|c|c|c|c|}
\hline \multicolumn{6}{|c|}{2017} & \multicolumn{6}{|c|}{2015} & \multirow{2}{*}{ بنود الانفاق } \\
\hline |المرونة & $\mathbf{P}$ & chi2 & R-Sq & Coef. & Cons. & المرونة & $\mathbf{P}$ & chi2 & R-Sq & Coef. & Cons. & \\
\hline \multirow[t]{3}{*}{0.72} & 0.00 & 858.7 & 0.98 & -0.012601 & 0.176620 & 0.74 & 0.00 & 272.6 & 0.93 & -0.010318 & 0.141372 & الحبوب والخبز \\
\hline & & & & -29.3 & 39.4 & & & & & -16.5 & 23.0 & $\mathbf{Z}$ \\
\hline & & & & 0.00 & 0.00 & & & & & 0.00 & 0.00 & $\mathbf{z}<\mathbf{P}$ \\
\hline \multirow[t]{3}{*}{0.96} & 0.00 & 57.9 & 0.75 & -0.004226 & 0.069003 & 0.96 & 0.00 & 61.5 & 0.75 & -0.003974 & 0.063912 & اللحوم \\
\hline & & & & -7.6 & 11.9 & & & & & -7.8 & 12.8 & $\mathbf{Z}$ \\
\hline & & & & 0.00 & 0.00 & & & & & 0.00 & 0.00 & $\mathbf{z}<\mathbf{P}$ \\
\hline \multirow[t]{3}{*}{0.27} & 0.00 & 110.5 & 0.85 & -0.018251 & 0.245702 & 0.25 & 0.00 & 286.0 & 0.93 & -0.018660 & 0.243289 & الأسماك \\
\hline & & & & -10.5 & 13.6 & & & & & -16.9 & 22.4 & $\mathbf{Z}$ \\
\hline & & & & 0.00 & 0.00 & & & & & 0.00 & 0.00 & $\mathbf{z}<\mathbf{P}$ \\
\hline \multirow[t]{3}{*}{0.71} & 0.00 & 557.5 & 0.97 & -0.016374 & 0.202582 & 0.80 & 0.00 & 1231.2 & 0.98 & -0.012210 & 0.148471 & الألبان والجبن والبيض \\
\hline & & & & -23.6 & 28.0 & & & & & -35.1 & 43.4 & $\mathbf{Z}$ \\
\hline & & & & 0.00 & 0.00 & & & & & 0.00 & 0.00 & $\mathbf{z}<\mathbf{P}$ \\
\hline \multirow[t]{3}{*}{0.90} & 0.00 & 162.9 & 0.90 & -0.003167 & 0.053441 & 0.87 & 0.00 & 116.9 & 0.85 & -0.003898 & 0.061251 & الزيوت والدهون \\
\hline & & & & -12.8 & 20.6 & & & & & -10.8 & 17.3 & $\mathbf{Z}$ \\
\hline & & & & 0.00 & 0.00 & & & & & 0.00 & 0.00 & $\mathbf{z}<\mathbf{P}$ \\
\hline \multirow[t]{3}{*}{\begin{tabular}{|l|}
-0.17 \\
\end{tabular}} & 0.00 & 1557.0 & 0.99 & -0.024052 & 0.302395 & 0.12 & 0.00 & 1120.7 & 0.98 & -0.020355 & 0.251994 & الفاكهة \\
\hline & & & & -39.5 & 47.6 & & & & & -33.5 & 42.2 & $\mathbf{Z}$ \\
\hline & & & & 0.00 & 0.00 & & & & & 0.00 & 0.00 & $\mathbf{z}<\mathbf{P}$ \\
\hline \multirow[t]{3}{*}{0.85} & 0.00 & 627.5 & 0.97 & -0.008066 & 0.102685 & 0.91 & 0.00 & 1068.5 & 0.98 & \begin{tabular}{|l|}
-0.004967 \\
\end{tabular} & 0.065770 & الخضر \\
\hline & & & & -25.1 & 30.6 & & & & & -32.7 & 44.1 & $\mathbf{Z}$ \\
\hline & & & & 0.00 & 0.00 & & & & & 0.00 & 0.00 & $\mathbf{z}<\mathbf{P}$ \\
\hline \multirow[t]{3}{*}{0.78} & 0.00 & 647.5 & 0.97 & -0.004161 & 0.051724 & 0.82 & 0.00 & 1253.4 & 0.98 & -0.003077 & 0.038316 & السكر والأغذية \\
\hline & & & & -25.5 & 30.3 & & & & & -35.4 & 44.9 & $\mathbf{Z}$ \\
\hline & & & & 0.00 & 0.00 & & & & & 0.00 & 0.00 & $\mathbf{z}<\mathbf{P}$ \\
\hline \multirow[t]{3}{*}{0.34} & 0.00 & 46.2 & 0.71 & -0.005619 & 0.075642 & 0.74 & 0.00 & 88.4 & 0.82 & -0.002087 & 0.034800 & المصنفة تحت بنود الأغية غير \\
\hline & & & & -6.8 & 8.8 & & & & & -9.4 & 16.0 & $\mathbf{Z}$ \\
\hline & & & & 0.00 & 0.00 & & & & & 0.00 & 0.00 & $\mathbf{z}<\mathbf{P}$ \\
\hline \multirow[t]{3}{*}{0.22} & 0.00 & 64.3 & 0.77 & -0.013425 & 0.241068 & -0.23 & 0.00 & 133.6 & 0.87 & -0.017664 & 0.280186 & المشروبات غير \\
\hline & & & & -8.0 & 13.8 & & & & & -11.6 & 18.7 & $\mathbf{Z}$ \\
\hline & & & & 0.00 & 0.00 & & & & & 0.00 & 0.00 & $\mathbf{z}<\mathbf{P}$ \\
\hline
\end{tabular}

المصدر: حسبت من بيانات الجهاز المركزى للتعبئة العامة و الاحصاء، بحث الدخل و الانفاق و الاستهلاك لعام 2015، سبتمبر 2016.

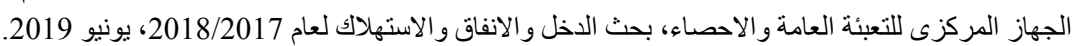

للجمهورية لعام 2017 بالمقارنة بعام 2015 (استثناء السكر و الاغذية السكرية) مما قد يشير الى أن هذه السلع أصبحت أكثر ضرورية.

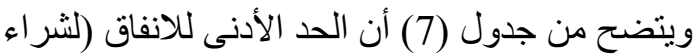

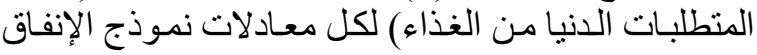

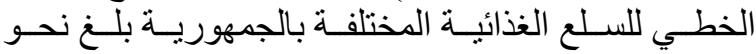
( 7809.6،

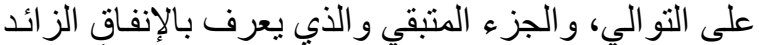

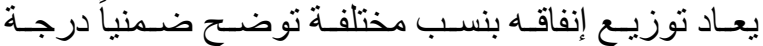

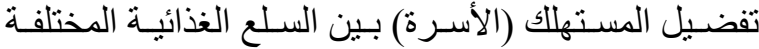
وذلك يتم وفقا لقيمة الميل الحدى لنسبة الإنفاق الاستهلاكي.

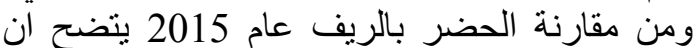

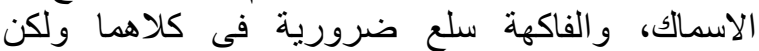

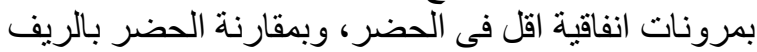

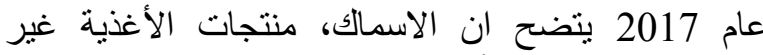

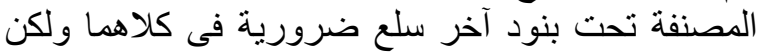

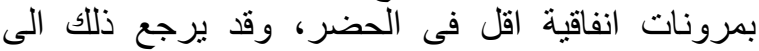
ارتفاع الحد الأدنى للانفاق فى الريف عن الحضر سواء
الميل الحدى لنسبة الإنفاق الاستهلاكي.

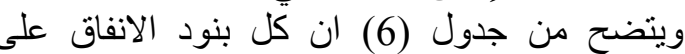

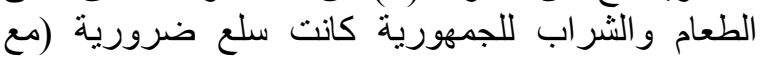
الاختلاف فى تصنيفها) باستنتاء المشروبات غير الفير الكحولية

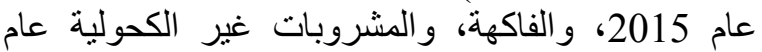
2017 (فهى سلع رديئة)، وقد تبين أن قيمة الأنة المرونة

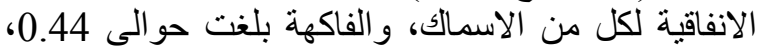

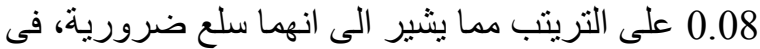

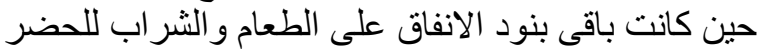

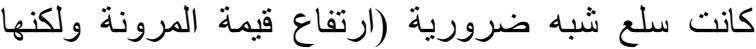

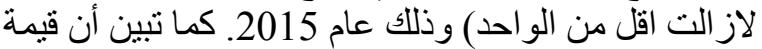
المرونة الانفاقية لكل من الاسماك، منتجات الحات الأغذية غير

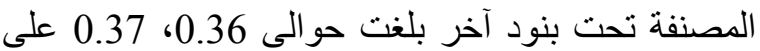

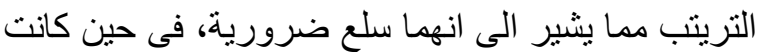

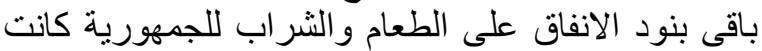

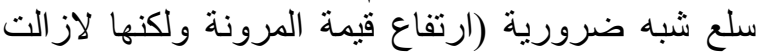

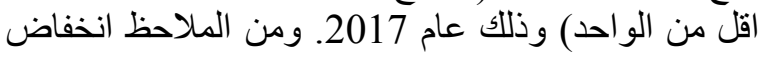
المرونة الانفاقية لمعظم بنود الانفاق على الطعام والثر اب الثماب 
جدول (3): نتائج نموذج LES لبنود الانفاق الغذائية للحضر لعام 2015، 2017.

\begin{tabular}{|c|c|c|c|c|c|c|c|c|c|c|}
\hline \multicolumn{5}{|c|}{2017} & \multicolumn{5}{|c|}{2015} & \multirow[b]{2}{*}{ بنود الانفاق } \\
\hline $\mathbf{P}$ & chi2 & R-Sq & المديل & الحدانالادنى & $\mathbf{P}$ & chi2 & R-Sq & الحديل & الحدانالادنى & \\
\hline \multirow[t]{3}{*}{0.00} & 109.2 & 0.85 & 0.0212 & 1556.4 & 0.00 & 66.8 & 0.77 & 0.0181 & 789.8 & الحبوب و الخبز \\
\hline & & & 10.5 & 10.7 & & & & 8.2 & 9.8 & $\mathbf{Z}$ \\
\hline & & & 0.00 & 0.00 & & & & 0.00 & 0.00 & $\mathbf{z}<\mathbf{P}$ \\
\hline \multirow[t]{3}{*}{0.00} & 35.2 & 0.65 & 0.0144 & 832.7 & 0.00 & 35.4 & 0.64 & 0.0166 & 409.1 & اللحوم \\
\hline & & & 8.2 & 6.6 & & & & 11.3 & 7.7 & $\mathbf{Z}$ \\
\hline & & & 0.00 & 0.00 & & & & 0.00 & 0.00 & $\mathbf{z}<\mathbf{P}$ \\
\hline \multirow[t]{3}{*}{0.00} & 91.2 & 0.83 & 0.0276 & 1833.5 & 0.00 & 72.3 & 0.78 & 0.0274 & 1153.7 & الأسماك \\
\hline & & & 9.6 & 8.9 & & & & 8.5 & 9.9 & $\mathbf{Z}$ \\
\hline & & & 0.00 & 0.00 & & & & 0.00 & 0.00 & $\mathbf{z}<\mathbf{P}$ \\
\hline \multirow[t]{3}{*}{0.00} & 35.2 & 0.65 & 0.0066 & 1258.4 & 0.00 & 35.4 & 0.64 & 0.0073 & 628.4 & الألبان والجبن والبيض \\
\hline & & & 5.9 & 15.7 & & & & 6.0 & 14.1 & $\mathbf{Z}$ \\
\hline & & & 0.00 & 0.00 & & & & 0.00 & 0.00 & $\mathbf{z}<\mathbf{P}$ \\
\hline \multirow[t]{3}{*}{0.00} & 234.8 & 0.93 & 0.0146 & 567.9 & 0.00 & 99.5 & 0.83 & 0.0149 & 385.7 & الزيوت والدهون \\
\hline & & & 15.3 & 8.3 & & & & 10.0 & 7.1 & $\mathbf{Z}$ \\
\hline & & & 0.00 & 0.00 & & & & 0.00 & 0.00 & $\mathbf{z}<\mathbf{P}$ \\
\hline \multirow[t]{3}{*}{0.00} & 9.3 & 0.33 & 0.0070 & 2219.3 & 0.00 & 20.3 & 0.50 & 0.0123 & 1188.1 & الفاكهة \\
\hline & & & 3.1 & 13.5 & & & & 4.5 & 12.0 & $\mathbf{Z}$ \\
\hline & & & 0.00 & 0.00 & & & & 0.00 & 0.00 & $\mathbf{z}<\mathbf{P}$ \\
\hline \multirow[t]{3}{*}{0.00} & 44.0 & 0.70 & 0.0054 & 710.6 & 0.00 & 105.5 & 0.84 & 0.0083 & 317.4 & الخضر \\
\hline & & & 6.6 & 12.2 & & & & 10.3 & 10.9 & $\mathbf{Z}$ \\
\hline & & & 0.00 & 0.00 & & & & 0.00 & 0.00 & $\mathbf{z}<\mathbf{P}$ \\
\hline \multirow[t]{3}{*}{0.00} & 44.8 & 0.70 & 0.0019 & 324.3 & 0.00 & 68.3 & 0.77 & 0.0027 & 168.0 & السكر والأغذية السكرية \\
\hline & & & 6.7 & 15.8 & & & & 8.3 & 14.1 & $\mathbf{Z}$ \\
\hline & & & 0.00 & 0.00 & & & & 0.00 & 0.00 & $\mathbf{z}<\mathbf{P}$ \\
\hline \multirow[t]{3}{*}{0.00} & 1041.5 & 0.98 & 0.0131 & 379.8 & 0.00 & 333.6 & 0.94 & 0.0119 & 188.3 & المصنفة تحت بنود الأذية غير آخر \\
\hline & & & 32.3 & 13.0 & & & & 18.3 & 8.0 & $\mathbf{Z}$ \\
\hline & & & 0.00 & 0.00 & & & & 0.00 & 0.00 & $\mathbf{z}<\mathbf{P}$ \\
\hline \multirow[t]{4}{*}{0.00} & 111.0 & 0.85 & 0.0644 & 3174.5 & 0.00 & 116.8 & 0.85 & 0.0689 & 1792.6 & المشروبات غير الكحولية \\
\hline & & & 10.5 & 7.2 & & & & 10.8 & 7.8 & $\mathbf{Z}$ \\
\hline & & & 0.00 & 0.00 & & & & 0.00 & 0.00 & $\mathbf{z}<\mathbf{P}$ \\
\hline & & & 0.1761 & 12857.6 & & & & 0.1882 & 7021.2 & الجملة بالجنيه \\
\hline
\end{tabular}

المصدر : حسبت من بيانات الجهاز المركزى للتعبئة العامة و الاحصاء، بحث الدخل والانفاق و الاستهلاك لعام 2015، سبتمبر 2016. الجهاز المركزى للتعبئة العامة و الاحصاء، بحث الدخل و الانفاق و الاستهلاك لعام 2018/2017، بونيو 2019.

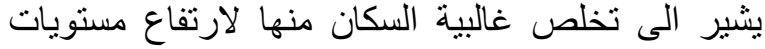
الانفاق المطلوبة. كما يتضح ارتفاعة النفان قيمة المرونة الانفاقية

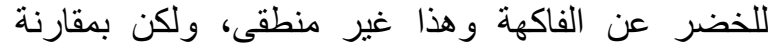

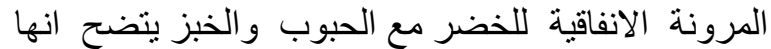

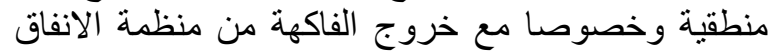
عام 2017.

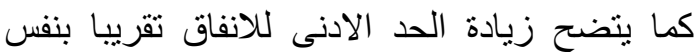

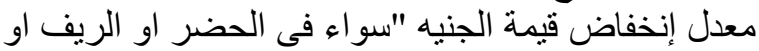

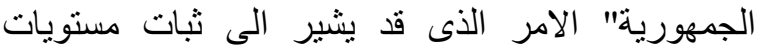
الانفاق لعام 2017 عند نفس المستوى لعام 2015.

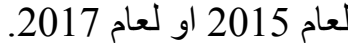
ومن العرض السابق بتضح عدم تمانشى بعض النتائج

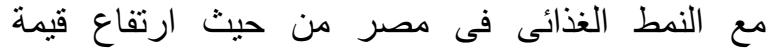
المرونات الانفاقية للحبوب والخبز (أهم المجموعات في المثية

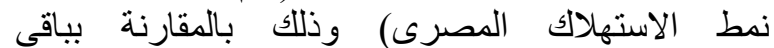
المجموعات، الامر الذى قد يعزى الى وجود أثر لسياسة

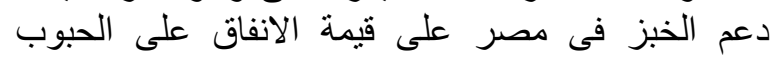

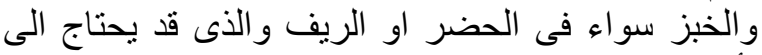

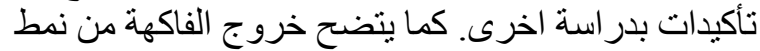
الاستهلاك المصرى (حضر وجمهورية) وفقا للاكية للمرونة السالبة عام 2017 باعتبار ها سلعة ردئية، والامر الذى قد 


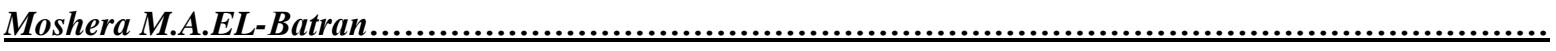

جلول (4): نتائج نموذج working لبنود الانفاق الغذائية للريف لعام 2015، 2017.

\begin{tabular}{|c|c|c|c|c|c|c|c|c|c|c|c|c|}
\hline \multicolumn{6}{|c|}{2017} & \multicolumn{6}{|c|}{2015} & \multirow[b]{2}{*}{ بنود الانفاق } \\
\hline |المرونة & $\mathbf{P}$ & chi2 & R-Sq & Coef. & Cons. & المرونة & $\mathbf{P}$ & chi2 & R-Sq & Coef. & Cons. & \\
\hline \multirow[t]{3}{*}{0.78} & 0.00 & 97.3 & 0.84 & -0.012115 & 0.178470 & 0.77 & 0.00 & 79.9 & 0.80 & -0.011200 & 0.157108 & الحبوب والخبز \\
\hline & & & & -9.9 & 14.2 & & & & & -8.9 & 12.9 & $\mathbf{Z}$ \\
\hline & & & & 0.00 & 0.00 & & & & & 0.00 & 0.00 & $\mathbf{z}<\mathbf{P}$ \\
\hline \multirow[t]{3}{*}{0.96} & 0.00 & 51.5 & 0.73 & -0.004735 & 0.074123 & 0.98 & 0.00 & 17.5 & 0.47 & -0.002850 & 0.052698 & 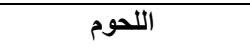 \\
\hline & & & & -7.2 & 11.0 & & & & & -4.2 & 8.0 & $\mathbf{Z}$ \\
\hline & & & & 0.00 & 0.00 & & & & & 0.00 & 0.00 & $\mathbf{z}<\mathbf{P}$ \\
\hline \multirow[t]{3}{*}{0.45} & 0.00 & 735.9 & 0.97 & -0.014315 & 0.192444 & 0.48 & 0.00 & 217.1 & 0.92 & -0.013143 & 0.178659 & الأسماك \\
\hline & & & & -27.1 & 35.7 & & & & & -14.7 & 20.7 & $\mathbf{Z}$ \\
\hline & & & & 0.00 & 0.00 & & & & & 0.00 & 0.00 & $\mathbf{z}<\mathbf{P}$ \\
\hline \multirow[t]{3}{*}{0.68} & 0.00 & 11083.8 & 1.00 & -0.015119 & 0.195787 & 0.73 & 0.00 & 898.0 & 0.98 & -0.013933 & 0.173601 & الألبان والجبن والبيض \\
\hline & & & & -105.3 & 133.3 & & & & & -30.0 & 38.5 & $\mathbf{Z}$ \\
\hline & & & & 0.00 & 0.00 & & & & & 0.00 & 0.00 & $\mathbf{z}<\mathbf{P}$ \\
\hline \multirow[t]{3}{*}{0.87} & 0.00 & 254.4 & 0.93 & -0.005440 & 0.079815 & 0.88 & 0.00 & 143.7 & 0.88 & -0.004735 & 0.072224 & الزيوت والدهون \\
\hline & & & & -16.0 & 22.9 & & & & & -12.0 & 18.9 & $\mathbf{Z}$ \\
\hline & & & & 0.00 & 0.00 & & & & & 0.00 & 0.00 & $\mathbf{z}<\mathbf{P}$ \\
\hline \multirow[t]{3}{*}{0.15} & 0.00 & 373.6 & 0.95 & -0.020867 & 0.275424 & 0.14 & 0.00 & 2043.5 & 0.99 & -0.022894 & 0.287151 & الفاكهة \\
\hline & & & & -19.3 & 24.9 & & & & & -45.2 & 58.5 & $\mathbf{Z}$ \\
\hline & & & & 0.00 & 0.00 & & & & & 0.00 & 0.00 & $\mathbf{z}<\mathbf{P}$ \\
\hline \multirow[t]{3}{*}{0.85} & 0.00 & 7011.9 & 1.00 & -0.009586 & 0.123930 & 0.89 & 0.00 & 119.2 & 0.86 & -0.006939 & 0.088185 & الخضر \\
\hline & & & & -83.7 & 105.9 & & & & & -10.9 & 14.3 & $\mathbf{Z}$ \\
\hline & & & & 0.00 & 0.00 & & & & & 0.00 & 0.00 & $\mathbf{z}<\mathbf{P}$ \\
\hline \multirow[t]{3}{*}{0.89} & 0.00 & 387.1 & 0.95 & -0.002992 & 0.039314 & 0.81 & 0.00 & 3136.9 & 0.99 & -0.004033 & 0.048255 & السكر والأغذية السكرية \\
\hline & & & & -19.7 & 25.3 & & & & & -56.0 & 69.2 & $\mathbf{Z}$ \\
\hline & & & & 0.00 & 0.00 & & & & & 0.00 & 0.00 & $\mathbf{z}<\mathbf{P}$ \\
\hline \multirow[t]{3}{*}{0.45} & 0.00 & 576.3 & 0.97 & -0.004949 & 0.066445 & 0.63 & 0.00 & 151.8 & 0.88 & -0.003460 & 0.048025 & المصنفة تحت بنود آخرى الأغية غير \\
\hline & & & & -24.0 & 31.5 & & & & & -12.3 & 17.6 & $\mathbf{Z}$ \\
\hline & & & & 0.00 & 0.00 & & & & & 0.00 & 0.00 & $\mathbf{z}<\mathbf{P}$ \\
\hline \multirow[t]{3}{*}{-0.99} & 0.00 & 200.4 & 0.91 & -0.032167 & 0.450107 & -1.32 & 0.00 & 34.8 & 0.64 & -0.033857 & 0.457799 & المشروبات غير الكحولية \\
\hline & & & & -14.2 & 19.4 & & & & & -5.9 & 8.2 & $\mathbf{Z}$ \\
\hline & & & & 0.00 & 0.00 & & & & & 0.00 & 0.00 & $\mathbf{Z}<\mathbf{P}$ \\
\hline
\end{tabular}

المصدر: حسبت من بيانات الجهاز المركزى للتعبئة العامة و الاحصاء، بحث الدخل و الانفاق و الاستهلاك لعام 2015 2015، سبتمبر 2016. الجهاز المركزى للتعبئة العامة و الاحصاء، بحث الدخل و الانفاق و الاستهلاك لعام 2018/2017، يونيو 2019. 


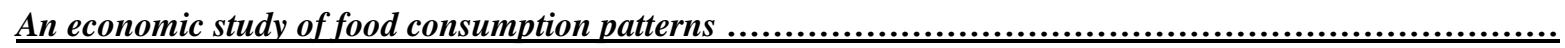

جدول (5): نتائج نموذج LES لبنود الانفاق الغذائية للريف لعام 2015، 2017.

\begin{tabular}{|c|c|c|c|c|c|c|c|c|c|c|}
\hline \multicolumn{5}{|c|}{2017} & \multicolumn{5}{|c|}{2015} & \multirow[b]{2}{*}{ بنود الانفاق } \\
\hline $\mathbf{P}$ & chi2 & R-Sq & الحدى الميل & للانفاق الانى & $\mathbf{P}$ & chi2 & R-Sq & الميل الحدى & للانفاق الانى & \\
\hline 0.00 & 31.7 & 0.63 & 0.0228 & 1933.1 & 0.00 & 45.2 & 0.69 & 0.0251 & 912.5 & الحبوب والخبز \\
\hline & & & 5.6 & 9.1 & & & & 6.7 & 8.8 & $\mathbf{Z}$ \\
\hline & & & 0.00 & 0.00 & & & & 0.00 & 0.00 & $\mathbf{z}<\mathbf{P}$ \\
\hline 0.00 & 54.6 & 0.74 & 0.0130 & 873.3 & 0.00 & 38.3 & 0.66 & 0.0183 & 403.1 & اللحوم \\
\hline & & & 5.7 & 7.4 & & & & 8.1 & 6.4 & $\mathbf{Z}$ \\
\hline & & & 0.00 & 0.00 & & & & 0.00 & 0.00 & $\mathbf{z}<\mathbf{P}$ \\
\hline 0.00 & 55.8 & 0.75 & 0.0221 & 1493.6 & 0.00 & 47.6 & 0.70 & 0.0277 & 937.8 & الأسماك \\
\hline & & & 7.5 & 9.6 & & & & 6.9 & 8.4 & $\mathbf{Z}$ \\
\hline & & & 0.00 & 0.00 & & & & 0.00 & 0.00 & $\mathbf{z}<\mathbf{P}$ \\
\hline 0.00 & 54.6 & 0.74 & 0.0166 & 1390.7 & 0.00 & 38.3 & 0.66 & 0.0155 & 765.6 & الألبان والجبن والبيض \\
\hline & & & 7.4 & 11.8 & & & & 6.2 & 11.1 & $\mathbf{Z}$ \\
\hline & & & 0.00 & 0.00 & & & & 0.00 & 0.00 & $\mathbf{z}<\mathbf{P}$ \\
\hline 0.00 & 109.9 & 0.85 & 0.0163 & 675.3 & 0.00 & 79.1 & 0.80 & 0.0184 & 423.3 & الزيوت والدهون \\
\hline & & & 10.5 & 8.3 & & & & 8.9 & 7.4 & $\mathbf{Z}$ \\
\hline & & & 0.00 & 0.00 & & & & 0.00 & 0.00 & $\mathbf{z}<\mathbf{P}$ \\
\hline 0.00 & 25.4 & 0.57 & 0.0185 & 2294.4 & 0.00 & 33.5 & 0.63 & 0.0251 & 1318.3 & الفاكهة \\
\hline & & & 5.0 & 11.9 & & & & 5.8 & 11.0 & $\mathbf{Z}$ \\
\hline & & & 0.00 & 0.00 & & & & 0.00 & 0.00 & $\mathbf{z}<\mathbf{P}$ \\
\hline 0.00 & 36.7 & 0.66 & 0.0094 & 906.7 & 0.00 & 37.9 & 0.65 & 0.0097 & 396.5 & الخضر \\
\hline & & & 6.1 & 11.2 & & & & 6.2 & 9.1 & $\mathbf{Z}$ \\
\hline & & & 0.00 & 0.00 & & & & 0.00 & 0.00 & $\mathbf{z}<\mathbf{P}$ \\
\hline 0.00 & 36.7 & 0.66 & 0.0028 & 313.1 & 0.00 & 31.5 & 0.61 & 0.0028 & 191.4 & السكر والأغذية السكرية \\
\hline & & & 6.1 & 12.7 & & & & 5.6 & 13.8 & $\mathbf{Z}$ \\
\hline & & & 0.00 & 0.00 & & & & 0.00 & 0.00 & $\mathbf{z}<\mathbf{P}$ \\
\hline 0.00 & 62.1 & 0.77 & 0.0086 & 485.9 & 0.00 & 106.9 & 0.84 & 0.0099 & 229.4 & المصنفة تحت بنود الأغذية غير \\
\hline & & & 7.9 & 8.6 & & & & 10.3 & 8.6 & $\mathbf{Z}$ \\
\hline & & & 0.00 & 0.00 & & & & 0.00 & 0.00 & $\mathbf{z}<\mathbf{P}$ \\
\hline 0.00 & 113.6 & 0.86 & 0.0830 & 3288.7 & 0.00 & 113.3 & 0.85 & 0.0887 & 2025.3 & المشروبات غير \\
\hline & & & 10.7 & 8.1 & & & & 10.6 & 8.8 & $\mathbf{Z}$ \\
\hline & & & 0.00 & 0.00 & & & & 0.00 & 0.00 & $\mathbf{z}<\mathbf{P}$ \\
\hline & & & 0.2131 & 13654.7 & & & & 0.2412 & 7603.2 & الجملة بالجنيه \\
\hline
\end{tabular}

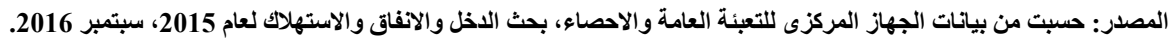
الجهاز المركزى للتعبئة العامة والاحصاء، بحث الدخل والانفاق والاستهلاك لعام 2018/2017، يونيو 2019. 


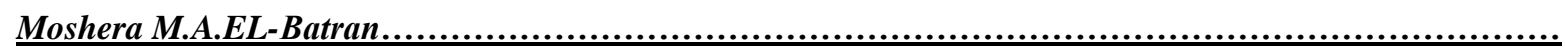

جدول (6): نتائج نموذج working لبنود الانفاق الغذائية للجمهورية لعام 2015، 2017.

\begin{tabular}{|c|c|c|c|c|c|c|c|c|c|c|c|c|}
\hline \multicolumn{6}{|c|}{2017} & \multicolumn{6}{|c|}{2015} & \multirow{2}{*}{ بنود الانفاق } \\
\hline المرونة & $\mathbf{P}$ & chi2 & R-Sq & Coef. & Cons. & المرونة & $\mathbf{P}$ & chi2 & R-Sq & Coef. & Cons. & \\
\hline \multirow[t]{3}{*}{0.72} & 0.00 & \begin{tabular}{|l|}
239.7 \\
\end{tabular} & 0.93 & -0.014043 & 0.194828 & 0.74 & 0.00 & 140.5 & 0.88 & -0.011554 & 0.157410 & الحبوب والخبز \\
\hline & & & & -15.5 & 20.6 & & & & & -11.9 & 16.5 & $\mathbf{Z}$ \\
\hline & & & & 0.00 & 0.00 & & & & & 0.00 & 0.00 & $\mathbf{z}<\mathbf{P}$ \\
\hline \multirow[t]{3}{*}{0.95} & 0.00 & 64.6 & 0.77 & -0.005243 & 0.079332 & 0.97 & 0.00 & 38.6 & 0.66 & -0.003303 & 0.057181 & اللحوم \\
\hline & & & & -8.0 & 11.7 & & & & & -6.2 & 11.0 & $\mathbf{Z}$ \\
\hline & & & & 0.00 & 0.00 & & & & & 0.00 & 0.00 & $\mathbf{z}<\mathbf{P}$ \\
\hline \multirow[t]{3}{*}{0.36} & 0.00 & 242.0 & 0.93 & -0.016005 & 0.216086 & 0.44 & 0.00 & 291.3 & 0.94 & -0.013901 & 0.190591 & الأسماك \\
\hline & & & & -15.6 & 20.2 & & & & & -17.1 & 24.0 & $\mathbf{Z}$ \\
\hline & & & & 0.00 & 0.00 & & & & & 0.00 & 0.00 & $\mathbf{z}<\mathbf{P}$ \\
\hline \multirow[t]{3}{*}{0.67} & 0.00 & 1434.0 & 0.99 & -0.016496 & 0.207081 & 0.75 & 0.00 & 1581.8 & 0.99 & -0.014068 & 0.171229 & الألبان والجبن والبيض \\
\hline & & & & -37.9 & 45.6 & & & & & -39.8 & 49.6 & $\mathbf{Z}$ \\
\hline & & & & 0.00 & 0.00 & & & & & 0.00 & 0.00 & $\mathbf{z}<\mathbf{P}$ \\
\hline \multirow[t]{3}{*}{0.85} & 0.00 & 163.3 & 0.90 & -0.005292 & 0.076833 & 0.86 & 0.00 & 228.8 & 0.92 & -0.004659 & 0.070293 & الزيوت والدهون \\
\hline & & & & -12.8 & 17.8 & & & & & -15.1 & 23.4 & $\mathbf{Z}$ \\
\hline & & & & 0.00 & 0.00 & & & & & 0.00 & 0.00 & $\mathbf{z}<\mathbf{P}$ \\
\hline \multirow[t]{3}{*}{-0.07} & 0.00 & 1013.1 & 0.98 & -0.023573 & 0.300531 & 0.08 & 0.00 & 2230.2 & \begin{tabular}{|l|}
0.99 \\
\end{tabular} & -0.022971 & 0.283322 & الفاكهة \\
\hline & & & & -31.8 & 38.9 & & & & & -47.2 & 59.6 & $\mathbf{Z}$ \\
\hline & & & & 0.00 & 0.00 & & & & & 0.00 & 0.00 & $\mathbf{z}<\mathbf{P}$ \\
\hline \multirow[t]{3}{*}{0.83} & 0.00 & 1505.0 & 0.99 & -0.009598 & 0.121619 & 0.90 & 0.00 & 1171.2 & 0.98 & -0.006194 & 0.079958 & الخضر \\
\hline & & & & -38.8 & 47.2 & & & & & -34.2 & 45.2 & $\mathbf{Z}$ \\
\hline & & & & 0.00 & 0.00 & & & & & 0.00 & 0.00 & $\mathbf{Z}<\mathbf{P}$ \\
\hline \multirow[t]{3}{*}{0.84} & 0.00 & 1682.4 & 0.99 & -0.003582 & 0.045564 & 0.82 & 0.00 & 1950.0 & 0.99 & -0.003604 & 0.043903 & السكر والأغذية \\
\hline & & & & -41.0 & 50.1 & & & & & -44.2 & 55.1 & $\mathbf{Z}$ \\
\hline & & & & 0.00 & 0.00 & & & & & 0.00 & 0.00 & $\mathbf{z}<\mathbf{P}$ \\
\hline \multirow[t]{3}{*}{0.37} & 0.00 & 122.6 & 0.87 & -0.005294 & 0.071186 & 0.71 & 0.00 & 112.1 & 0.85 & -0.002601 & 0.039956 & المصنجة ات الأغذية غير \\
\hline & & & & -11.1 & 14.3 & & & & & -10.6 & 16.7 & $\mathbf{Z}$ \\
\hline & & & & 0.00 & 0.00 & & & & & 0.00 & 0.00 & $\mathbf{z}<\mathbf{P}$ \\
\hline \multirow[t]{3}{*}{-0.74} & 0.00 & 182.9 & 0.91 & -0.028372 & 0.403662 & -0.66 & 0.00 & 331.8 & 0.94 & -0.024306 & 0.354475 & المشروبات غير \\
\hline & & & & -13.5 & 18.5 & & & & & -18.2 & 27.2 & $\mathbf{Z}$ \\
\hline & & & & 0.00 & 0.00 & & & & & 0.00 & 0.00 & $\mathbf{Z}<\mathbf{P}$ \\
\hline
\end{tabular}

المصدر: حسبت من بيانات الجهاز المركزى للتعبئة العامة و الاحصاء، بحث الدخل و الانفاق و الاستهلاك لعام 2015، 2015، سبتمبر 2016.

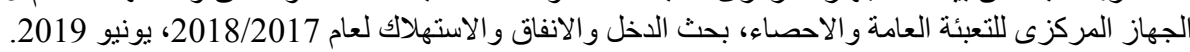


جدول (7): نتائج نموذج LES لبنود الانفاق الغذائية للجمهورية لعام 2015، 2017.

\begin{tabular}{|c|c|c|c|c|c|c|c|c|c|c|}
\hline \multicolumn{5}{|c|}{2017} & \multicolumn{5}{|c|}{2015} & \multirow[b]{2}{*}{ بنود الانفاق } \\
\hline $\mathbf{P}$ & chi2 & R-Sq & الحدى & للانفاثى الحث & $\mathbf{P}$ & chi2 & $\begin{array}{l}\text { R- } \\
\text { Sq }\end{array}$ & الميل الحدى & للانفاتى الحد & \\
\hline \multirow[t]{3}{*}{0.00} & 28.8 & 0.60 & 0.0149 & 1961.4 & 0.00 & 47.2 & 0.70 & 0.0182 & 920.7 & الحبوب والخبز \\
\hline & & & 5.4 & 9.1 & & & & 6.9 & 10.2 & $\mathbf{Z}$ \\
\hline & & & 0.00 & 0.00 & & & & 0.00 & 0.00 & $\mathbf{z}<\mathbf{P}$ \\
\hline \multirow[t]{3}{*}{0.00} & 19.9 & 0.51 & 0.0098 & 982.8 & 0.00 & 21.5 & 0.52 & 0.0166 & 423.0 & 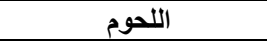 \\
\hline & & & 5.1 & 6.5 & & & & 10.3 & 7.7 & $\mathbf{Z}$ \\
\hline & & & 0.00 & 0.00 & & & & 0.00 & 0.00 & $\mathbf{z}<\mathbf{P}$ \\
\hline \multirow[t]{3}{*}{0.00} & 33.6 & 0.64 & 0.0191 & 1856.5 & 0.00 & 70.2 & 0.78 & 0.0279 & 1038.0 & الأسماك \\
\hline & & & 5.8 & 7.2 & & & & 8.4 & 9.1 & $\mathbf{Z}$ \\
\hline & & & 0.00 & 0.00 & & & & 0.00 & 0.00 & $\mathbf{z}<\mathbf{P}$ \\
\hline \multirow[t]{3}{*}{0.00} & 19.9 & 0.51 & 0.0061 & 1464.0 & 0.00 & 21.5 & 0.52 & 0.0077 & 767.2 & الألبان والجبن والبيض \\
\hline & & & 4.5 & 13.6 & & & & 4.6 & 13.6 & $\mathbf{Z}$ \\
\hline & & & 0.00 & 0.00 & & & & 0.00 & 0.00 & $\mathbf{z}<\mathbf{P}$ \\
\hline \multirow[t]{3}{*}{0.00} & 37.4 & 0.66 & 0.0102 & 781.0 & 0.00 & 85.9 & 0.81 & 0.0149 & 440.5 & الزيوت والدهون \\
\hline & & & 6.1 & 6.0 & & & & 9.3 & 8.0 & $\mathbf{Z}$ \\
\hline & & & 0.00 & 0.00 & & & & 0.00 & 0.00 & $\mathbf{z}<\mathbf{P}$ \\
\hline \multirow[t]{3}{*}{0.00} & 9.3 & 0.33 & 0.0070 & 2402.8 & 0.00 & 16.2 & 0.45 & 0.0130 & 1360.8 & الفاكهة \\
\hline & & & 3.1 & 13.5 & & & & 4.0 & 12.4 & $\mathbf{Z}$ \\
\hline & & & 0.00 & 0.00 & & & & 0.00 & 0.00 & $\mathbf{z}<\mathbf{P}$ \\
\hline \multirow[t]{3}{*}{0.00} & 21.1 & 0.53 & 0.0043 & 893.6 & 0.00 & 68.6 & 0.77 & 0.0079 & 392.0 & الخضر \\
\hline & & & 4.6 & 12.3 & & & & 8.3 & 12.0 & $\mathbf{Z}$ \\
\hline & & & 0.00 & 0.00 & & & & 0.00 & 0.00 & $\mathbf{z}<\mathbf{P}$ \\
\hline \multirow[t]{3}{*}{0.00} & 20.5 & 0.52 & 0.0015 & 343.0 & 0.00 & 55.8 & 0.74 & 0.0026 & 185.4 & السكر والأغذية السكرية \\
\hline & & & 4.5 & 13.2 & & & & 7.5 & 15.8 & $\mathbf{Z}$ \\
\hline & & & 0.00 & 0.00 & & & & 0.00 & 0.00 & $\mathbf{z}<\mathbf{P}$ \\
\hline \multirow[t]{3}{*}{0.00} & 40.5 & 0.68 & 0.0084 & 516.5 & 0.00 & 305.8 & 0.94 & 0.0114 & 207.3 & المصنفة تحت بنود آخير \\
\hline & & & 6.4 & 5.0 & & & & 17.5 & 9.3 & $\mathbf{Z}$ \\
\hline & & & 0.00 & 0.00 & & & & 0.00 & 0.00 & $\mathbf{z}<\mathbf{P}$ \\
\hline \multirow[t]{4}{*}{0.00} & 34.0 & 0.64 & 0.0474 & 4009.5 & 0.00 & 99.9 & 0.83 & 0.0694 & 2074.6 & المشروبات غير الكحولية \\
\hline & & & 5.8 & 6.3 & & & & 10.0 & 8.8 & $\mathbf{Z}$ \\
\hline & & & 0.00 & 0.00 & & & & 0.00 & 0.00 & $\mathbf{z}<\mathbf{P}$ \\
\hline & & & 0.1286 & 15211.1 & & & & 0.1896 & 7809.6 & الجملة بالجنيه \\
\hline
\end{tabular}

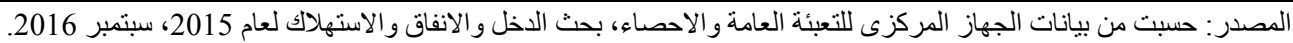

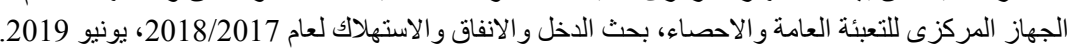

عبـداللطيف، عـزت محمـود (2017). التقدير الاحصـائى الإنى

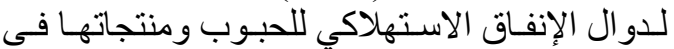

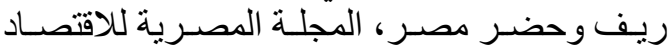
الزراعي، 27 (4): ديسمبر (ب)، دوب، 2205-214. القبلاوي عبدربه ، مصطفي وعلي محمود السيد، هبة الله

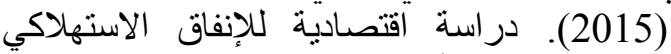

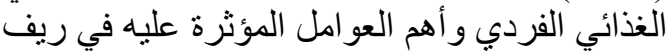

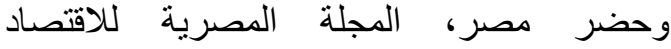
الزر اعي، 25، (2): يونيو، 691، 696-706.

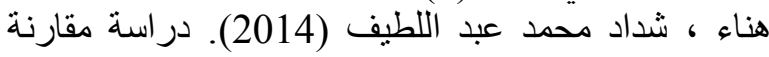

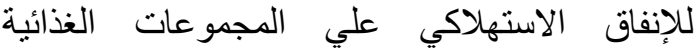
الرئيسية في ريف وحضر مصرئ الانيفي المجلة المصرية

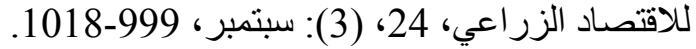
وفاء ، عبد الكريم محمد حسين (2015). التفين التحليل الاقتصادي لهيكل الإنفاق الغذائي للأسرة المصرينة الأيرة،

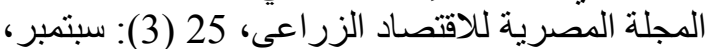

$.950-937$

\section{6. - المراجع العربية}

نخال عبدالحمبد عبدالحمبد ، جمال (2013). در اسة تحليلية للإنفاق الاستهلاكي الغذائي الفردي في في ريف

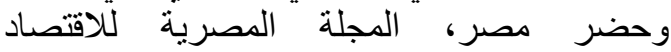
الزراعي، 23 (1):، مارس، معزئ 319-319.

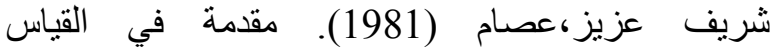
الإقتصادى، دار الطليعة للطباعة و النشر ، بيروت، لبعة لبنان. لميس ،فوزي البهنسي مصطفي (2012). دراسة اقتصادية دارية للأنماط الإنفاقية الاستهلاكية المصرية، ركاية رسالة

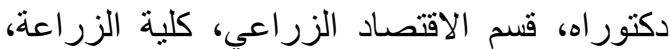
جامعة المنصورة فيورة العر اقى، بدير محمد و السبد حسين سرحان، حسين (2017).

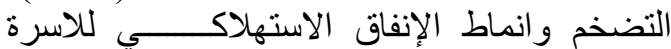

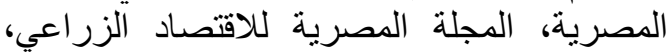

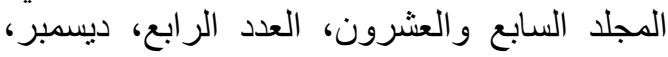
1900-1883 


\section{EFERENCES}

Barten A.P. (1964). Consumer Demand Functions under Conditions of Almost Additive Preferences, Econometrica. Vol. $32: 1-38$.

Deaton A. and Muellbauer, J. (1984). Economics and Consumer Behavior. Cambridge University Press, New York, USA.

Perthel D. (1995). Engel's Law Revisited, Int. Stal. Rev. 43 :211-213

Seale J. L. Jr., Regmi, A. and Bernstein, J. (2003), International Evidence on Food Consumption Patterns, Electronic Report from the Economic Research Service, United States Department of Agriculture, Economic Research Service/USDA, Tech. Bull. NO. 1904, October.
Stone R. (1964). Linear Expenditure System and Demand Analysis: An Application to the pattern of British Demand, Econ. J., 64:511-527.

Theil H. and Clements K. W. (1987). Applied Demand Analysis: Results from SystemWide Approaches. Ballinger Publishing Company, Cambridge, MA, USA.

Theil H., Chung C. F. and Seale J.L. (1989). International Evidence on Consumption Patterns. JAI Press, Inc., Greenwich, CT.

Theil H. (1965). The Information Approach to Demand Analysis, Econometrica, 33 :6787.

Working H. (1943) .Statistical Laws of Family Expenditure, J. Amer. Stat. Asso.38, (1):43-56 